\title{
Experimental studies on damage detection in frame structures using vibration measurements
}

\author{
Giancarlo Fraraccio $^{\mathrm{a}}$, Adrian Brügger ${ }^{\mathrm{b}, *}$ and Raimondo Betti ${ }^{\mathrm{c}}$ \\ ${ }^{a}$ Università degli Studi di Roma La Sapienza, Dipartimento di Ingegneria Strutturale e Geotecnica, Via \\ Eudossiana, 18, 00184 Roma, Italy \\ ${ }^{\mathrm{b}}$ Department of Civil Engineering and Engineering Mechanics, Columbia University, 636A S. W. Mudd Building, \\ 500 West 120th Street, New York, NY, USA \\ ${ }^{\mathrm{c}}$ Department of Civil Engineering and Engineering Mechanics, Columbia University, 640 S. W. Mudd Building, \\ 500 West 120th Street, New York, NY, USA
}

Received 2 August 2007

Revised 4 August 2009

\begin{abstract}
This paper presents an experimental study of frequency and time domain identification algorithms and discusses their effectiveness in structural health monitoring of frame structures using acceleration input and response data. Three algorithms were considered: 1) a frequency domain decomposition algorithm (FDD), 2) a time domain Observer Kalman IDentification algorithm (OKID), and 3) a subsequent physical parameter identification algorithm (MLK). Through experimental testing of a four-story steel frame model on a uniaxial shake table, the inherent complications of physical instrumentation and testing are explored. Primarily, this study aims to provide a dependable first-order and second-order identification of said test structure in a fully instrumented state. Once the characteristics (i.e. the stiffness matrix) for a benchmark structure have been determined, structural damage can be detected by a change in the identified structural stiffness matrix. This work also analyzes the stability of the identified structural stiffness matrix with respect to fluctuations of input excitation magnitude and frequency content in an experimental setting.
\end{abstract}

Keywords: System identification, damage detection, health monitoring, ERA, OKID, FDD, experimental, shake table, vibration

\section{Introduction}

System identification can be described as the definition of a mathematical or numerical model that attempts to represent the behavior of a physical phenomenon or system to an acceptable degree of precision. From the perspective of a structural engineer, the physical system is generally a structure that can be defined by physical characteristics such as mass, damping, and stiffness. However, such physical characteristics can rarely be measured directly. It is therefore more common to measure the modal properties such as natural frequencies, damping ratios, and mode shapes; this is the outcome of most frequency domain based methodologies. In the context of time domain analysis, the goal is to find an operator that accurately maps a given dynamic input on the system to its measured response, allowing the user to predict the system's response to arbitrary future inputs.

In civil engineering and especially in earthquake engineering applications, the measurement of a structure's acceleration response proves significantly more economical and less intrusive than the quantification of, for example,

${ }^{*}$ Corresponding author. Tel.: +1 212854 3143; Fax: +1 212854 6267; E-mail: adrian@ civil.columbia.edu. 
displacement or local strain. Today, accelerometer and data acquisition technology is sufficiently mature that the commercial products available to the researcher are not only reliable and lightweight, but also quite economical. This type of equipment lends itself well to permanent as well as temporary instrumentation. Consequently, the use of acceleration instrumentation in civil engineering has become quite common, as has the research regarding system identification with acceleration time history data.

The classical approach to dynamic system identification relies on the identification of the system's modal parameters to subsequently update an approximate initial finite element (FEM) model of the system. This approach has been given exhaustive attention by Ewins [1], Mottershead and Friswell [2], Berman [3], Baruch [4], and Beck and Katafygiotis [5]. A more direct approach, the direct identification of modal parameters from dynamic data has also been discussed by Agbabian et al. [6] and Smyth et al. [7].

With increased computing capabilities, numerical methods in the time domain have become increasingly popular. The transformation of the second-order differential equation of motion of a structure to a system of first-order equations provides a computationally efficient framework for identifying a system in the time domain. This area has been studied in depth by Ibrahim and Mikulcik [8], Ibrahim [9], and Vold et al. [10]. The Eigensystem Realization Algorithm (ERA) [11,12] by Juang and Pappa provides the basis of many modern time-domain identification algorithms. It could be argued that the most notable derivative of the ERA is the Observer Kalman filter IDentification (OKID) algorithm, pioneered by Juang et al. [13] and applied extensively by Lu" et al. [14,15].

Different approaches have been proposed to obtain the physical parameters of a system, i.e. mass, stiffness, and damping, using the identified modal properties. The transformation of the damped modal vectors to their secondorder undamped state has been studied by Sestieri and Ibrahim [16] and applied extensively by Tseng et al. [17]. Classical assumptions of uncoupled modes have led to the standard technique of modal identification, as shown by Imregun and Ewins [18], Ibrahim [19], and Alvin [20].

The complex eigenvectors gained from the state-space identification must first be transformed to the real form to gain a physical meaning. An approach which uses the complex eigenvectors gained through the OKID identification technique to solve for the physical parameters of the system - mass, stiffness, and damping matrices - has been proposed by De Angelis et al. [21]. In this work the minimum requirement for sensor/actuator distribution has also been optimized. This physical parameter identification approach will be referred to as the MLK algorithm.

Another approach to system identification is provided by frequency domain identification. The Frequency Domain Decomposition (FDD), as performed by Brincker et al. [22,23], provides a modal identification of the system: using this algorithm, natural frequencies, modal damping ratios, and a good estimation of the physical mode shapes can be obtained. A significant strength of Frequency Domain Decomposition lies in the fact that it is an output-only algorithm, requiring no input to provide a dependable identification of the structure, and it is capable of accurately extracting the damping of closely spaced modes. The main limitation of this identification algorithm is that its identified mode shapes are not orthogonal. However, the intuitive nature of the identification technique makes it a very powerful tool for the identification of a structure in the benchmark phase.

The aim of this paper is to explore the effectiveness of these algorithms (OKID, MLK, and FDD) in an experimental setting by using a physical structural model on a uniaxial shake table. The susceptibility of these approaches to real physical interference, measurement error, signal noise, etc. is the main focus of this work. The variance of the identified parameters of special interest, as it is a measure of the health monitoring scheme's minimal successful damage detection threshold.

\section{Algorithm definitions}

\subsection{FDD algorithm}

The Frequency Domain Decomposition (FDD) is closely linked to basic Frequency Domain techniques, such as peak picking techniques. In such an approach, it is easy to obtain good estimates of the system's frequencies. However with closely spaced modes, it may be difficult to accurately identify the power of each mode and, consequently to obtain reliable damping factors. As previously mentioned, the frequency domain approach requires only the system's dynamic response for identification. 
The FDD retains the classical frequency approach's advantages; the intermediate data presented to the user is generally intuitive. The user must manually select the dominant peaks, as in classical peak picking techniques, ignoring anomalies from interference or input excitation leakage. Through a Singular Value Decomposition (SVD), the FDD effectively decomposes the $n$-DOF system into $n$ SDOF systems [22]. Each peak represents one SDOF system which can in turn be transformed into the time domain by inverse FFT. Once in the time domain, each SDOF mode's frequency and modal damping can be found from the autocorrelation function, i.e. IFFT of that peak.

The Power Spectral Density (PSD) at discrete frequencies $\omega_{i}$ for the $m$ measured responses $y(t)$ due to the $r$ inputs $u(t)$ can be written as:

$$
G_{y y}\left(\omega_{i}\right)=H\left(\omega_{i}\right) G_{u u} H\left(\omega_{i}\right)^{T}
$$

where $H(m \times r)$ is the frequency response function and the overbar and superscript $T$ indicate respectively the complex conjugate and the transpose. $G_{u u}\left(\omega_{i}\right)$ is the PSD of the $r$ unknown inputs, constant with respect to frequency for the case of white noise input. In that case, it can be shown that $G_{y y}\left(\omega_{i}\right)$ does not depend on the input but only on the frequency response.

The FDD creates a matrix $F_{i j}$ in which each column $j$ contains the FFT of $j$-th time history $(j=1,2, \ldots, m)$ and $i$ is the frequency index, $\omega_{i}$. The output PSD, $G_{y y}\left(\omega_{i}\right)$, is the cross-correlation of each row of $F_{i j}$ and can be decomposed at discrete frequencies through a singular value decomposition as:

$$
G_{y y}\left(\omega_{i}\right)=\bar{V}_{i} S V_{i}^{T}
$$

where $S_{i}=\operatorname{diag}\left[\begin{array}{lllll}s_{i 1} & s_{i 2} & \ldots & s_{i m}\end{array}\right]$ contains the singular values and $V_{i}=\left[\begin{array}{llll}v_{i 1} & v_{i 2} & \ldots & v_{i m}\end{array}\right]$ contains the respective singular vectors.

When the frequency $\omega_{i}$ is close to a structural mode, i.e. when there is a peak in the plot of the singular values, the first singular value (by convention of the SVD, the largest singular value in the spectrum) becomes dominant and reaches a local maximum at $\omega_{a}$. Consequently, the respective singular vector $\phi_{a}$ is assumed to be an accurate estimate of the real mode shape (i.e. the measured mode shape). All the singular values in the spectrum that are associated with the chosen mode shape are taken as part of the PSD of the SDOF representing the mode, i.e. the power in the frequency spectrum with the same singular vector is isolated into the SDOF peak. To test if a singular value obtained through Eq. (2) at frequencies $\omega_{i}$ belongs to the same mode, the respective singular vectors $v_{i j}, j=1$, $2, \ldots, m$ are compared through the Modal Assurance Criterion. The Modal Assurance Criterion (MAC) compares the linear dependence of the assumed real mode shape, $\phi_{a}$, and each singular vector, $v_{i j}$, in the spectrum and is defined as follows [24]:

$$
\operatorname{MAC}\left(\phi_{a}, v_{i j}\right)=\begin{gathered}
\left(\phi_{a}^{T} \cdot v_{i j}\right)^{2} \\
\left(\phi_{a}^{T} \cdot \phi_{a}\right)\left(v_{i j}^{T} \cdot v_{i j}\right)
\end{gathered}
$$

If the MAC between the estimated mode shape $\phi_{a}$ and a singular vector $v_{i j}$ is higher than a fixed limit, the corresponding singular value is chosen as part of the PSD of the SDOF. The procedure is repeated for all the selected peaks. The fixed limit of the MAC value is defined by the user to ensure that a sufficient number of singular values has been chosen to adequately isolate the power of each mode. It is important to mention that only the singular vector at the peak, $\phi_{a}$, is considered an acceptable estimation of the measured mode shape corresponding to natural frequency $\omega_{a}$; the singular vectors surrounding the peak $\left(v_{i j}\right)$ will become less parallel as the distance in $\omega$ from the peak increases. If located appreciably far away from a peak, a singular vector $v_{i j}$ may have no physical significance, as it merely represents an arbitrary member of a set of singular vectors which describe the system's $\operatorname{PSD}, G_{y y}\left(\omega_{i}\right)$.

The SDOF spectrum of isolated singular values for each peak is then transformed back to the time domain by inverse FFT. The resulting free decay time histories are used to obtain the modal frequencies by averaging the crossing times, and damping coefficients by calculating the logarithmic decrement [23].

The SDOF decomposition yields exact results if (i) the unknown input is white noise, (ii) the system is lightly damped and (iii) different mode shapes are geometrically orthogonal. For civil structures subjected to earthquake and wind excitation, the assumption of light damping is acceptable while the input excitation is characterized by a narrow-banded spectrum. The algorithm may fail to properly isolate modes during peak-picking if the measured modes shape vectors are not orthogonal. If two geometrically different structural modes provide similar measured mode shapes, the algorithm cannot distinguish between the two singular vectors and fail to isolate the two modes from each other.

In light of extending this algorithm to a real-time application, it should be noted that the calculation of the PSD matrices as well as the SVD of the PSD is computationally intensive. 


\subsection{OKID algorithm}

An alternative approach to identify structural parameters is to obtain a minimal realization of the system using input/output data in the time domain. One of the most important advantages in using a time domain approach is that it is possible to avoid errors due to transformation from time to frequency domain and vice versa. The aim of this section is to explain how to extract modal parameters from input/output measurements using the ERA/OKID algorithm.

Consider the equation of motion for a generic linear $n$-DOF system excited by $r$ external forces $u(t)$. These equations can be written in a second order formulation as:

$$
M \ddot{x}(t)+L \dot{x}(t)+K x(t)=B_{u} u(t)
$$

where $M, L, K$ are the mass, damping, and stiffness matrices respectively; $x(t)$ is a vector containing the $n$ nodal displacements; $\dot{()}$ and $\ddot{()}$ denote the first and second-order time derivatives, respectively; and $B_{u}$ is a matrix which allocates the external inputs $u(t)$ to the system's DOFs.

By introducing a state vector $z(t)=\left[x(t)^{T} \dot{x}(t)^{T}\right]^{T}$, it is possible to rewrite the equation of motion (4) in a first order formulation:

$$
\begin{aligned}
\dot{z}(t) & =A z(t)+B u(t) \\
y(t) & =C z(t)+D u(t)
\end{aligned}
$$

where

$$
A=\left[\begin{array}{cc}
0 & I \\
-M^{-1} K & -M^{-1} L
\end{array}\right],{ }_{B}=\left[\begin{array}{c}
0 \\
B_{u}
\end{array}\right],
$$

$C$ and $D$ represent a state-space description of the system in a continuous time formulation, while $y(t)$ is a vector of $m$ recorded responses. The matrix $A$ contains the modal information of the system, from which it is possible to obtain the complex eigenvalues and eigenvectors, respectively:

$$
\begin{aligned}
& \Lambda_{2 n \times 2 n}=\left[\begin{array}{llll}
\lambda_{1} & \lambda_{2} & \ldots & \lambda_{2 n}
\end{array}\right] \\
& \Phi_{2 n \times 2 n}=\left[\begin{array}{llll}
\phi_{1} & \phi_{2} & \ldots & \phi_{2 n}
\end{array}\right]
\end{aligned}
$$

while the $C$ and $D$ matrices depend on the type of measurement available (displacement, velocities and accelerations).

One representation of the system (one set of matrices $A, B, C$ and $D$ ) can be determined just by input-output mapping. Assuming that the system is controllable and observable, a first-order representation of the system can be obtained just using a set of input and output time histories, independent of the number of degrees-of-freedom of the structural model. Among the most popular techniques, the one based on Eigensystem Realization Algorithm with Data Correlation (ERA/DC) provides valid identification results. The system input and output matrices can be obtained from the Singular Value Decomposition of the Hankel matrix containing the input-output correlation functions. A significant improvement of the ERA methodology, in terms of both computation as well as accuracy, is represented by the Observer Kalman filter Identification (OKID: Juang et al. [13]). In this approach, the Markov parameters of a fictitious observer are first determined and then used to retrieve the system's Markov parameters. Such parameters are then included in the Hankel matrix and used to obtain a first order system representation. Hence, using the ERA/DC approach with OKID, the input-output mapping for a first order system can be expressed as:

$$
\begin{aligned}
& \dot{z}(t)=A_{i d} z(t)+B_{i d} u(t) \\
& y(t)=C_{i d} z(t)+D_{i d} u(t)
\end{aligned}
$$

where the matrices $A_{i d}, B_{i d}, C_{i d}$ and $D_{i d}$ represent the identified matrices. Solving the system's eigenproblem expressed by $A_{i d}$, it is possible to determine the system's eigenvalues $\left(\Lambda_{2 n \times 2 n}=\left[\begin{array}{llll}\lambda_{1} & \lambda_{2} & \ldots & \lambda_{2 n}\end{array}\right]\right)$ and the corresponding eigenvectors, from which frequencies and damping factors are easily retrieved. For details, the reader is referred to the work by Juang et al. [12,13], and Luş et al. [14]. 


\subsection{MLK identification}

An approach to determine the full-order mass, damping and stiffness matrices of the system is to start from the identified first order state space model of the structure and derive, through a set of transformations, the corresponding mass, damping and stiffness matrices. One of these methodologies (De Angelis et al. [21]) determines such matrices using the complex eigenvalues and corresponding complex eigenvectors of the associated damped eigenvalue problem:

$$
\left(\lambda_{i}^{2} M+\lambda_{i} L+K\right) \psi_{i}=0
$$

where $\psi_{i}(i=1,2, \ldots, 2 \mathrm{~N})$ represents the $i$-th complex eigenvector. If no rigid body modes are considered, the eigenvalues and the associated eigenvectors appear in complex conjugate pairs. These complex eigenvalues and eigenvectors can be uniquely determined by applying proper transformations to the identified state, input and output matrices of the state space model [21].

The system's complex eigenvectors can be scaled in order to satisfy equations [16].

$$
\begin{gathered}
\Theta^{T} \cdot\left[\begin{array}{cc}
L & M \\
M & 0
\end{array}\right] \cdot \Theta=I \\
\Theta^{T} \cdot\left[\begin{array}{cc}
K & 0 \\
0 & -M
\end{array}\right] \cdot \Theta=\Lambda
\end{gathered}
$$

where $\Theta=\left[\psi^{T}(\psi \Lambda)^{T}\right]^{T}$ and $\psi_{n \times 2 n}=\left[\psi_{1} \psi_{2} \cdots \psi_{2 n}\right]$, so that the mass, damping and stiffness matrices of the structure can be expressed as functions of the complex eigenvalues and eigenvectors:

$$
\begin{aligned}
M & =\left(\psi \cdot \Lambda \cdot \psi^{T}\right)^{-1} \\
K & =\left(\psi \cdot \Lambda^{-1} \cdot \psi^{T}\right)^{-1} \\
L & =-M\left(\psi \cdot \Lambda^{2} \cdot \psi^{T}\right) \cdot M
\end{aligned}
$$

If the forcing term $u(t)$ acting on the system is represented by an known arbitrary force applied to any arbitrary degree of freedom, then, if the measurement setup satisfies some completeness conditions [21], all 3 matrices can be easily obtained. However, in the case of external excitation as motion of the support, then the forcing term is dependent on the mass matrix and this affects the scaling of the eigenvectors and, consequently, the identification of the system's matrices $M, L$, and $K$. In the case of imposed support displacement, the transformation matrix correlating the different system cannot be uniquely determined, unless the value of one discrete mass is known. Hence, for the experimental phase of this study, since the input excitation will be in the form of imposed base displacements, the mass matrix will be assumed to be known.

\section{Experimental approach}

In this study the abovementioned algorithms are applied to experimental data gained from a four-story structural steel model (Fig. 1). All numerical computations in this study were performed in Matlab version 6.5.

\subsection{Experimental setup}

The structural model is mounted on an ANCO uniaxial hydraulic shake table with a $1.5 \mathrm{~m}$ by $1.5 \mathrm{~m}$ platform. The table's primary displacement and secondary acceleration feedback allows the simulation of arbitrary acceleration time histories in the frequency range of DC to $150 \mathrm{~Hz}$ at a peak ground acceleration of $3 \mathrm{~g}$.

The structure consists of four stories, with an interstory height of $533 \mathrm{~mm}$ and floor dimensions of $610 \mathrm{~mm}$ by $457 \mathrm{~mm}$. The structure is braced diagonally in one direction. The columns and bracing are manufactured from narrow structural steel plate stock, oriented such that the weak direction of bending is in the direction of excitation of the shake table. Consequently, the structure is preferentially flexible in the direction of excitation, allowing for a clear spectrum of bending modes in this direction. All structural connections are bolted, allowing fast exchange and 
Table 1

Data acquisition scheme

$\begin{array}{cll}\text { CH } & \text { Sensor Position } & \text { Orientation } \\ 1 & \text { Table Reference } & \text { Weak } \\ 2 & \text { 1st Floor } & \text { Weak } \\ 3 & \text { 2nd Floor } & \text { Weak } \\ 4 & \text { 3rd Floor } & \text { Weak } \\ 5 & \text { 4th Floor } & \text { Weak } \\ 6 & \text { 3rd Floor } & \text { Strong } \\ 7 & \text { 4th Floor } & \text { Strong } \\ 8 & \text { 4th Floor } & \text { Weak }\end{array}$

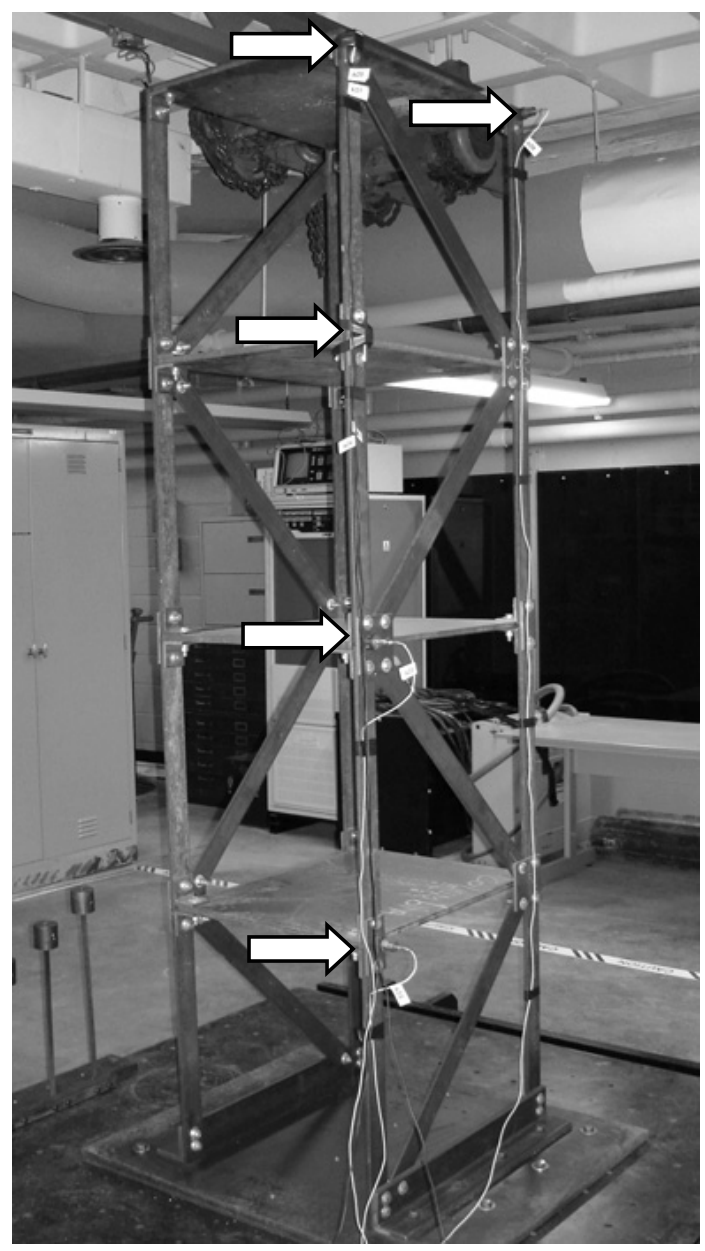

Fig. 1. Model with accelerometer positions shown by arrows.

modification of structural members. Hereafter, this direction of excitation shall be referred to as the weak bending direction and the direction perpendicular to excitation will be referred to as the strong bending direction of the structure.

The model is fully instrumented with piezoelectric accelerometers, with seven channels of acceleration response on the structural model and one reference channel on the shake table's platform. The sensors are attached magnetically to the column connection plates at each floor, at the height of the respective floor plate. The data acquisition scheme is outlined in Table 1 .

Even though no direct excitation occurs in the strong direction of the structure (i.e. perpendicular to direction of 
Table 2

Input acceleration time histories

$\begin{array}{cll}\text { Case \# } & \text { Earthquake } & \text { PGA/Run \# } \\ 1 & \text { El Centro } & 0.47 \mathrm{~g} \# 1 \\ 2 & \text { El Centro } & 0.47 \mathrm{~g} \mathrm{\# 2} \\ 3 & \text { El Centro } & 0.47 \mathrm{~g} \mathrm{\# 3} \\ 4 & \text { Hachinohe } & 0.27 \mathrm{~g} \# 1 \\ 5 & \text { Hachinohe } & 0.27 \mathrm{~g} \mathrm{\# 2} \\ 6 & \text { Hachinohe } & 0.27 \mathrm{~g} \mathrm{\# 3} \\ 7 & \text { Kobe } & 0.29 \mathrm{~g} \mathrm{\# 1} \\ 8 & \text { Kobe } & 0.29 \mathrm{~g} \# 2 \\ 9 & \text { Kobe } & 0.29 \mathrm{~g} \# 3 \\ 10 & \text { Northridge } & 0.43 \mathrm{~g} \# 1 \\ 11 & \text { Northridge } & 0.43 \mathrm{~g} \# 2 \\ 12 & \text { Northridge } & 0.43 \mathrm{~g} \# 3 \\ 13 & \text { Random noise } & 0.19 \mathrm{~g} \# 1 \\ 14 & \text { Random noise } & 0.19 \mathrm{~g} \mathrm{\# 2} \\ \mathbf{1 5} & \text { Random noise } & 0.19 \mathrm{~g} \mathrm{\# 3}\end{array}$

excitation of the table), sensors 6 and 7 do provide information on the torsional and strong bending modes in that direction. Channel 8 is placed on a second column on the 4th floor to further aid in the identification of torsional modes. All dynamic data is acquired at $256 \mathrm{~Hz}$. The steel structure mounted on the shake table is shown in Fig. 1, arrows indicating sensor positions.

\subsection{Simulation of damage}

In order to simulate a realistic damage scenario, i.e. the reduction of stiffness of a member in a structure, one column between the 2 nd and 3rd floor of the structure is replaced with members of smaller cross-section. Two damage cases were investigated. Damage Case One (DC1) provides a 66\% area reduction of the column, effecting a $22.2 \%$ stiffness reduction between the two floors in the weak bending direction. Since this reduction of stiffness is quite significant, a damage scenario with a lesser stiffness reduction was also introduced. Damage Case Two (DC2) provides a 33\% reduction in area, inducing a $13.9 \%$ reduction in stiffness in the weak direction. Hereafter, the undamaged case will be referred to as DC0. In the calculation of this theoretical stiffness reduction, the diagonal bracing is assumed to provide no bending stiffness in the weak direction, while the floor plates are assumed to be rigid.

In this experiment, it was found that the tightness of all structural bolts is critical for a precise identification of the structure's stiffness. As a consequence, bolts were tightened after every table run to guarantee the precision of the recorded data.

\subsection{Time-history inputs}

The following earthquake acceleration time histories were used in this test sequence: El Centro (1940), Hachinohe (1983), Northridge (1994), and Kobe (1995). In addition to the earthquake input, a thirty second time history of band-limited white noise with a frequency range of $1 \mathrm{~Hz}-80 \mathrm{~Hz}$ was also used. In order to minimize errors due to uncontrolled variables, every test was repeated three times. Table 2 shows a complete list of all 15 test runs with corresponding run numbers.

Considering that the structure's fundamental frequency is near $3.75 \mathrm{~Hz}$, a time scaling factor of $1 / \sqrt{ } 3$ was introduced to ensure that the structure was excited by the proper range of the time histories' power spectra. Furthermore, to prevent yielding and additional unexpected damage, the input time histories were scaled in magnitude to avoid excessive excitation of the structure.

Since the shake table control system invariably adds noise to the input time histories, the actual acceleration time histories measured by the table reference accelerometer are considered as the input for the system identification. Figures 2 and 3 allow a comparison between the Fast Fourier Transforms of the reference input time histories and the actual table excitation response, respectively. The shake table's response acceleration FFT shows a significant 

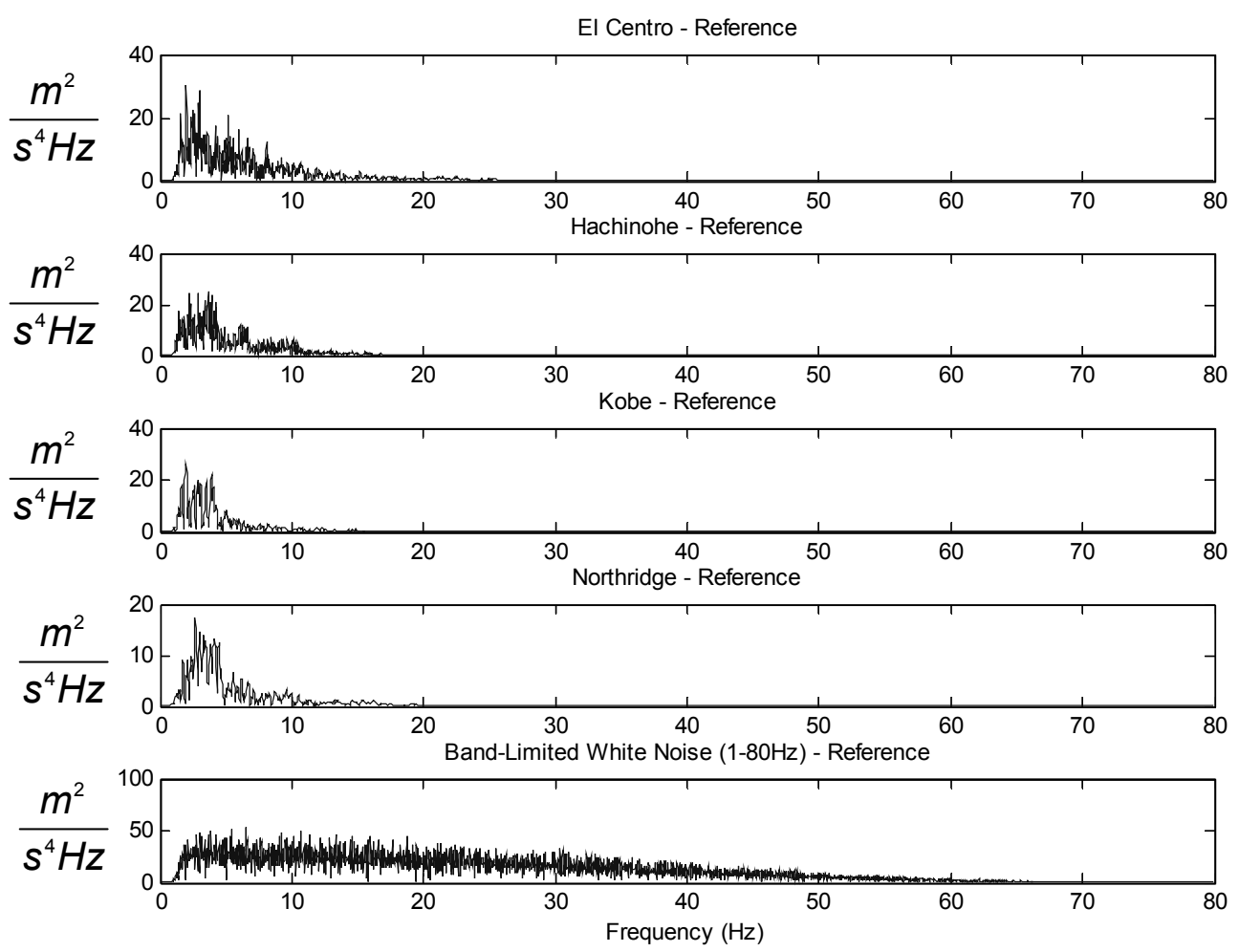

Fig. 2. FFT of reference input acceleration time histories.

amount of high-frequency noise, with tight-banded $60 \mathrm{~Hz}$ interference in all excitation time histories, a common problem with control systems operating on alternating current, and therefore an issue that must be considered in experimental and field monitoring projects. Efforts were made to isolate and shield the analog data lines to minimize this interference. However, the displacement controller of the shake table was determined to be the source of the majority of the interference. Even though the $60 \mathrm{~Hz}$ interference is relatively strong, it should not significantly alter the behavior of the structure since the interfence is very tightly banded and no structural mode is present in the vicinity of $60 \mathrm{~Hz}$ (e.g. all significant structural modes are below $30 \mathrm{~Hz}$, as proven by the numerical model and by analysis of the measured data).

\section{Numerical model}

\subsection{Model parameters}

In order to validate the proposed approaches, a simple four DOF FEM model was analyzed in Matlab. The structure was modelled as a shear-type steel structure with masses lumped at each floor. For each column, stiffness parameters were evaluated using $k_{i}={ }_{h^{3}}^{12 E I}$, where $E$ is the Young's modulus of structural steel, $I$ is the column's second moment of area in the direction of excitation, and $h$ is the floor height. The physical parameters of stiffness and mass are, respectively,

$$
K=\left[\begin{array}{cccc}
3.40 & -1.70 & 0.00 & 0.00 \\
-1.70 & 3.40 & -1.70 & 0.00 \\
0.00 & -1.70 & 3.40 & -1.70 \\
0.00 & 0.00 & -1.70 & 1.70
\end{array}\right](N / m)
$$


Table 3

Exact modal quantities of numerical model

$\begin{array}{cll}\text { Mode } & \text { Frequency }(\mathrm{Hz}) & \text { Damping }(\%) \\ 1 & 3.75 & 5.00 \\ 2 & 10.78 & 3.00 \\ 3 & 16.53 & 1.00 \\ 4 & 20.27 & 1.00\end{array}$
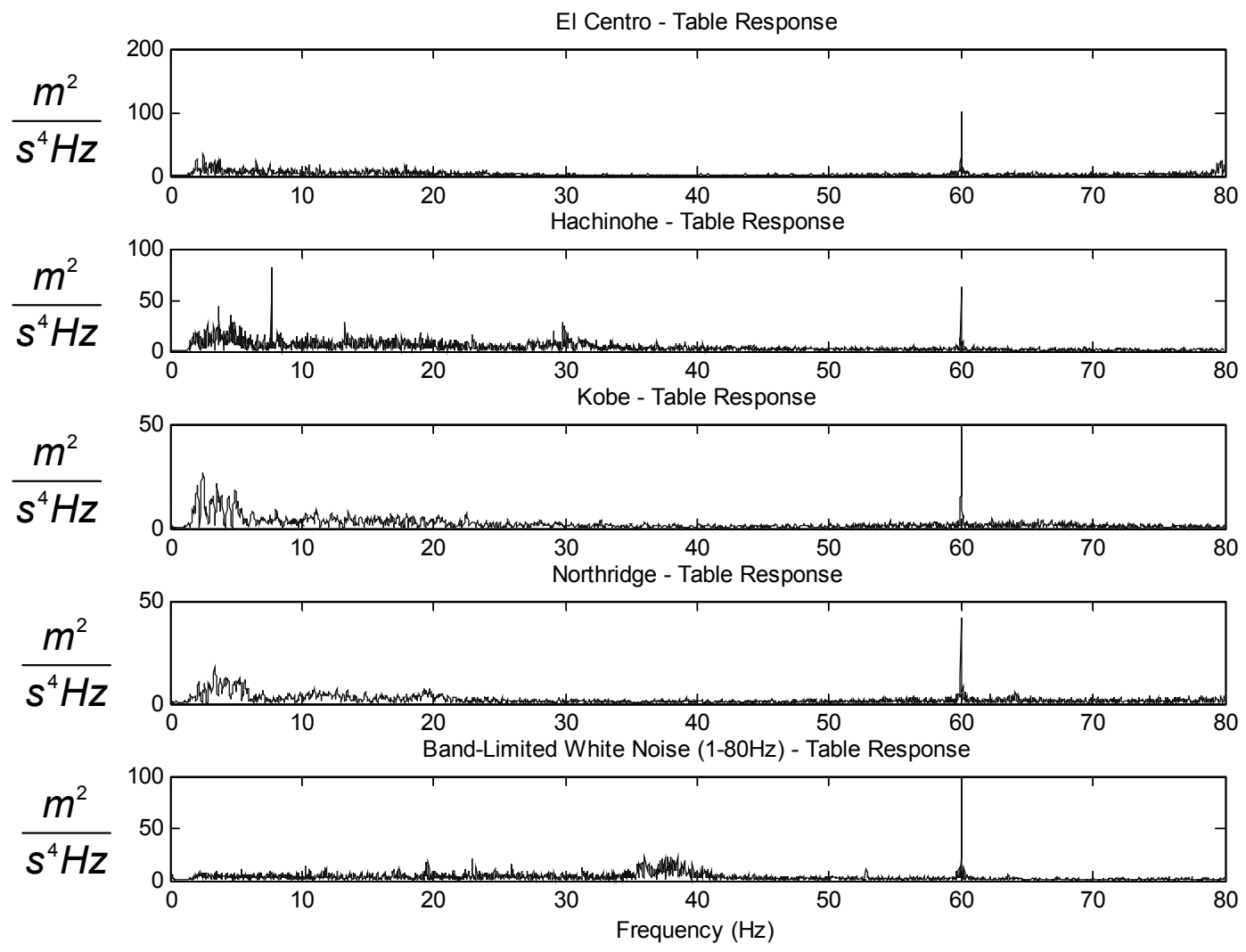

Fig. 3. FFT of Actual shake table acceleration response time histories.

$$
M=\left[\begin{array}{cccc}
37.00 & 0.00 & 0.00 & 0.00 \\
0.00 & 37.00 & 0.00 & 0.00 \\
0.00 & 0.00 & 37.00 & 0.00 \\
0.00 & 0.00 & 0.00 & 37.00
\end{array}\right](\mathrm{kg})
$$

with corresponding undamped natural frequencies and damping ratios, as shown in Table 3.

Using the Matlab toolbox Simulink, the numerical model was excited by the El Centro earthquake acceleration time history with a PGA of $0.20 \mathrm{~g}$. The response time histories gained from this numerical model serve as a benchmark to validate the effectiveness of both the FDD and the OKID/MLK algorithms.

\subsection{FDD identification}

A Frequency Domain Decomposition identification was performed on the response data from the numerical simulation to identify the numerical model's modal parameters. When extracting the singular values from the PSD of the four outputs considered, four peaks appear clearly, as shown in Fig. 4. 


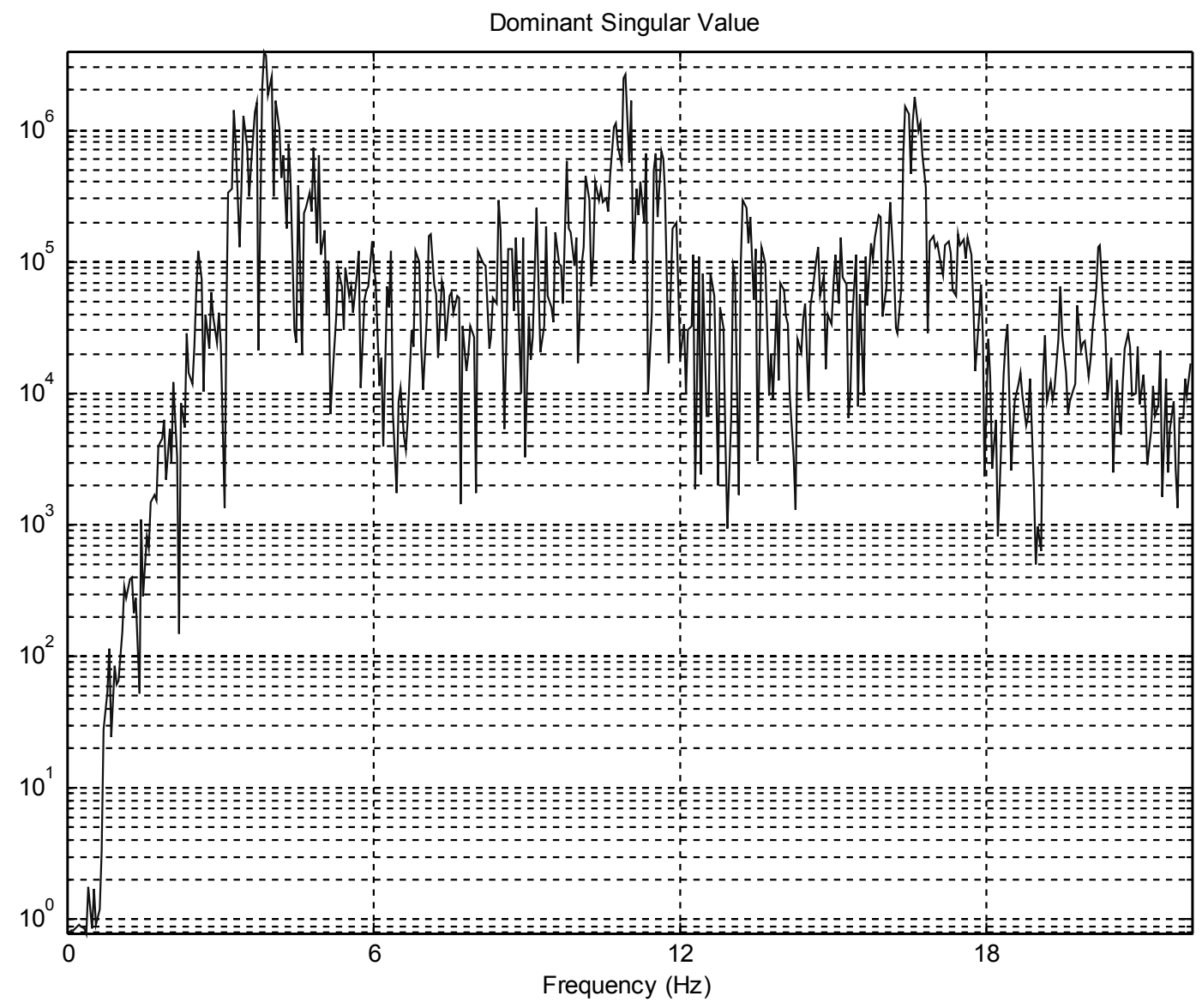

Fig. 4. Dominant singular value spectrum of numerical model (FDD).

In the FDD algorithm, the MAC value is chosen by the user to properly isolate the spectral power of each peak. In this case a MAC value of 0.9 was chosen to isolate the peaks completely. The choice of the MAC is a function of the degree of geometric orthogonality of the identified mode shapes. The user must choose a MAC that is small enough to isolate the entire power of the peak without including the power of any adjacent peaks with similar singular vectors, which would overestimate the damping of the mode. If a higher MAC is chosen, the user risks identifying only part of the peak's tail, consequently underestimating the damping of the structural mode being isolated.

Once the peaks are isolated, the resulting four free decay time histories can be extracted by IFFT (Fig. 5). For each of the four peaks, the singular vector corresponding to the dominant singular value can be taken as a good approximation of the real mode shape. The mode shapes in Fig. 6 can be identified as, from left to right: 1) the first weak bending mode, 2) the second weak bending mode, 3) the third weak bending mode, and 4) fourth weak bending mode. Comparing the identified mode shapes with the mode shapes obtained from the structural properties of the FEM model, it can be concluded that the FDD successfully identifies the mode shapes of the numerical model. In this case these mode shapes are essentially orthogonal; however, this may not be the case in general. Since the algorithm can only determine the measured mode shape, i.e. the relative magnitude in oscillation between sensor points, the structure must be adequately instrumented in order for all mode shapes to be identified with sufficient detail.

Using the free decay time histories in Fig. 5, the natural frequencies and damping ratios are calculated by estimating the crossing times and logarithmic decrement, respectively (Table 4a). Comparing these results with Table 3 shows that the identified frequencies are relatively accurate, with errors ranging from $2.67 \%$ for the first natural frequency to $0.25 \%$ for the fourth frequency. However, the identified damping ratios show significant error of up to $37 \%$; it is noteworthy that all identified values underestimate the actual modal damping of the system. 
Table 4

Identified modal quantities of numerical model by FDD (a) and OKID (b)

$\begin{array}{ccc}\text { Mode } & \text { Frequency }(\mathrm{Hz}) & \text { Damping }(\%) \\ 1 & 3.85 & 3.6 \\ 2 & 10.94 & 1.9 \\ 3 & 16.60 & 0.6 \\ 4 & 20.22 & 0.8\end{array}$

(a)

$\begin{array}{ccc}\text { Mode } & \text { Frequency }(\mathrm{Hz}) & \text { Damping }(\%) \\ 1 & 3.75 & 5.00 \\ 2 & 10.78 & 2.90 \\ 3 & 16.55 & 1.00 \\ 4 & 20.28 & 1.00\end{array}$

(b)

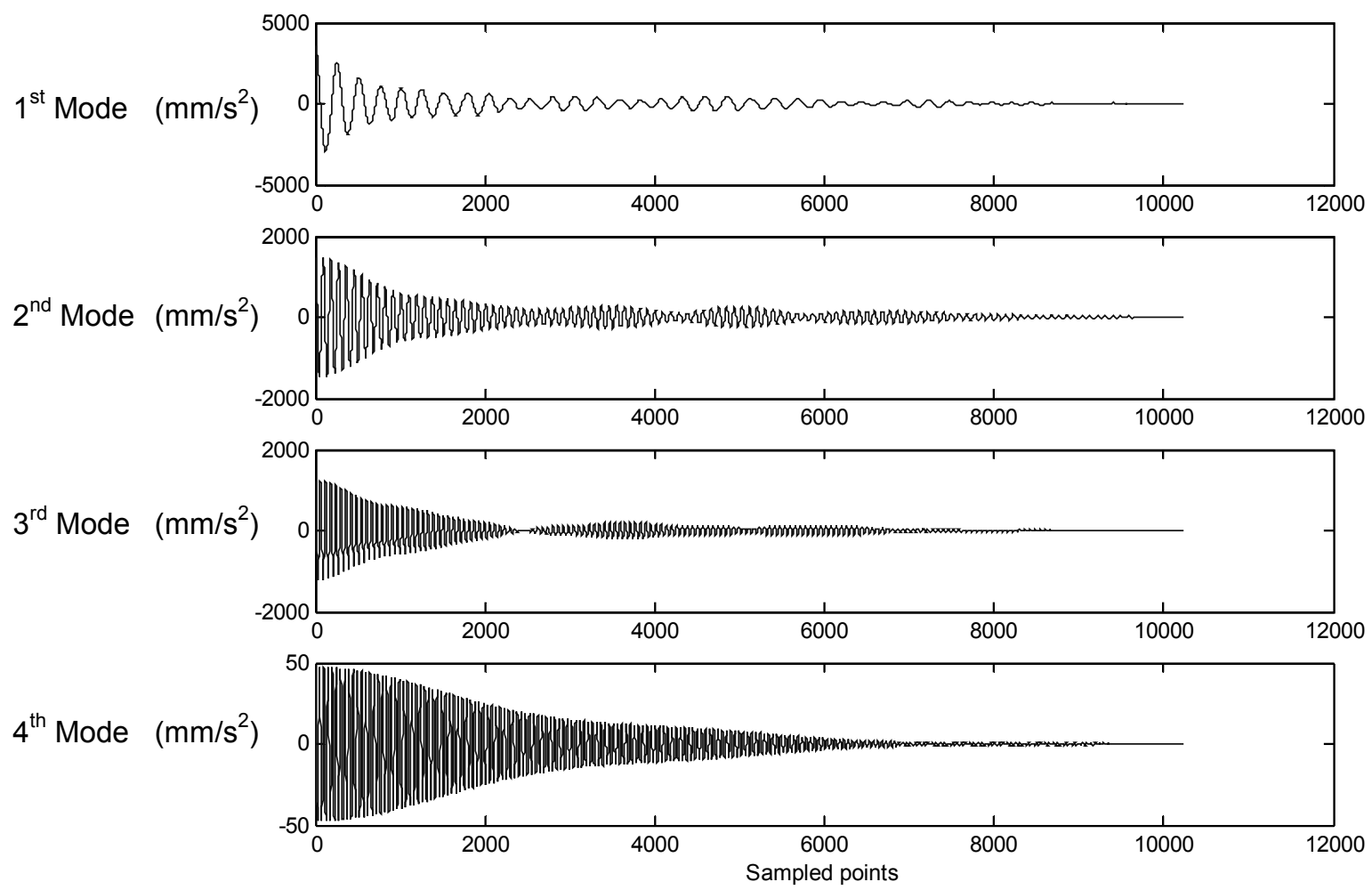

Fig. 5. Time-domain SDOF decay histories (FDD).

This identification depends also on the resolution available in the frequency domain, $d \omega=\underset{d t \cdot N}{1}$, where $d t$ is the sample time and $N$ is the number of sampled points. Zero-padding to 10,000 data points became necessary when only 30 seconds of dynamic data were available; this provides the necessary resolution for the identified frequencies but also increases the computational load significantly.

\subsection{OKID identification}

The time histories of the simulated input and of the structural response were also used to identify the numerical model with the ERA/OKID algorithm. This algorithm provides the modal parameters of the state space system matrix 

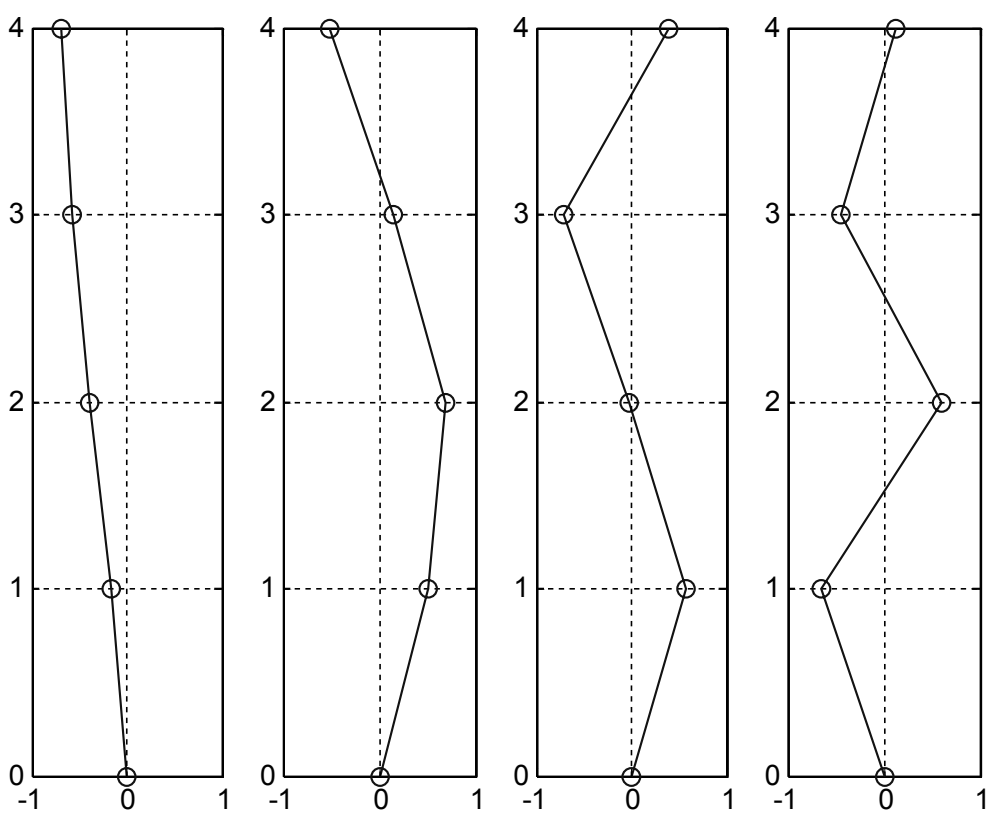

Fig. 6. Identified physical mode shapes (FDD).

A, (including the complex eigenvectors of the system) from which it is then possible to obtain the natural frequencies and damping ratios of the physical system, if certain assumptions are satisfied [14]. The natural frequencies and damping ratios of the physical system are shown in Table $4 \mathrm{~b}$; the complex eigenvectors will be used in the next section for the physical parameter (MLK) identification.

It is apparent that the modal frequencies and damping ratios identified by the OKID algorithm are much closer to the exact values than the parameters identified by FDD. The maximum error for the identified natural frequencies and damping ratios is a mere $0.0012 \%$ and $0.33 \%$, respectively.

\subsection{MLK identification}

Using the modal parameters and complex mode shapes from the OKID identification, a physical parameter identification was performed, assuming that the diagonal mass matrix is known:

$$
K_{\text {EXACT }}=\left[\begin{array}{cccc}
3.40 & -1.70 & 0.00 & 0.00 \\
-1.70 & 3.40 & -1.70 & 0.00 \\
0.00 & -1.70 & 3.40 & -1.70 \\
0.00 & 0.00 & -1.70 & 1.70
\end{array}\right](\mathrm{N} / \mathrm{m})
$$

In order to test the response of this approach to mass estimation error, a case with $15 \%$ mass error was also considered:

$$
K_{E 15}=\left[\begin{array}{cccc}
3.91 & -1.95 & 0.00 & 0.00 \\
-1.95 & 3.91 & -1.95 & 0.00 \\
0.00 & -1.95 & 3.91 & -1.95 \\
0.00 & 0.00 & -1.95 & 1.95
\end{array}\right](N / m)
$$

Even though the identified stiffness is skewed due to the mass estimation error, the identified stiffness still represents the global structure to an acceptable degree. In structural health monitoring the mass of a structure is estimated in the definition of the benchmark structure and generally remains unchanged after damage has occurred. Consequently, the identified stiffness matrix may be skewed with respect to the real stiffness matrix, but it will remain stable since the estimated mass matrix remains constant once it has been defined; therefore, the stiffness matrix still serves as a good indicator of damage. This robustness of the stiffness matrix with respect to mass estimation error allows 


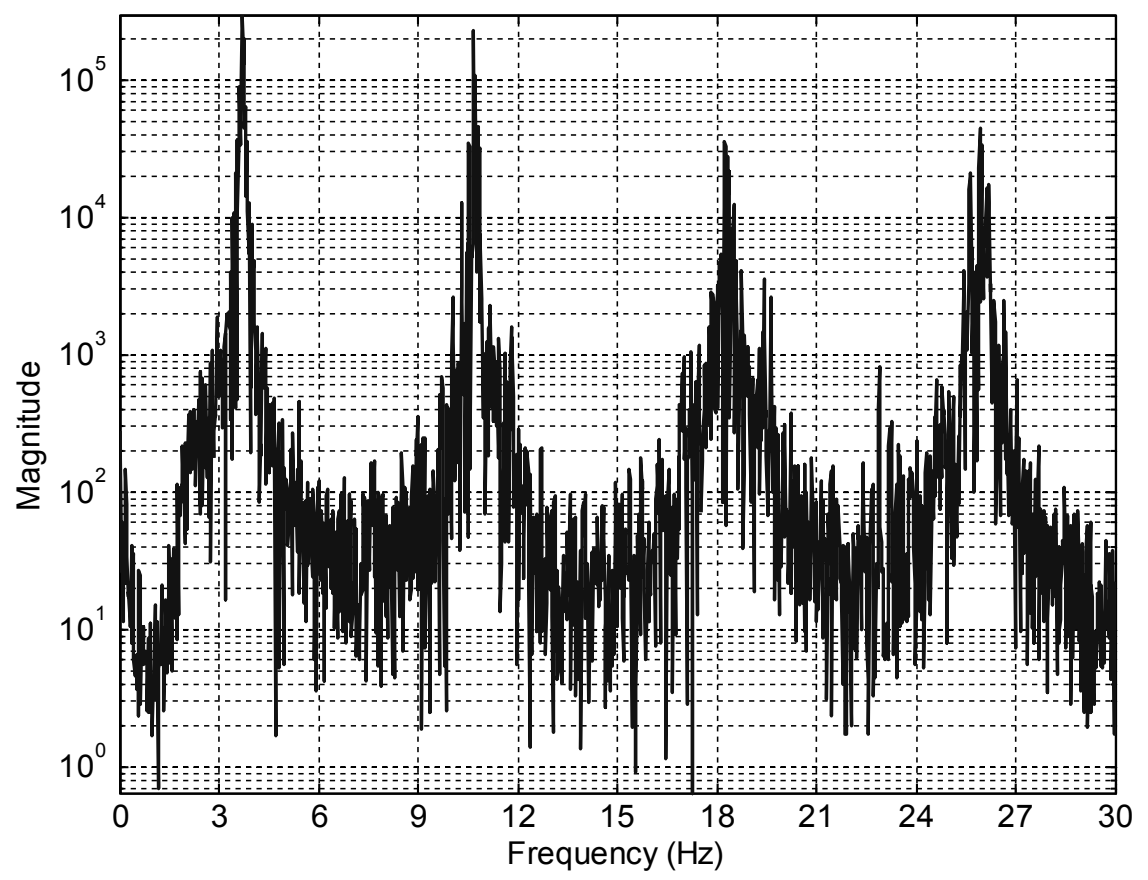

Fig. 7. Dominant singular value for output PSD, DC0 (FDD).

the proposed procedure to be used even if the structure's masses are not known exactly. Having validated both the FDD and OKID algorithms with a known numerical model, the physical test structure will now be identified using dynamic data gathered in an experimental setting.

\section{Damage identification}

In order to provide a dependable damage identification scheme, a structure must first be identified in the undamaged or benchmark state. Once damage occurs, a deviation in the dynamic behavior of the structure with respect to the benchmark allows a damage identification algorithm to identify the presence and, depending on the power of the algorithm and the availability of data on the structure, the location of damage. For damage identification, the structural model was tested on the shake table using the previously listed excitation cases.

\subsection{FDD identification}

The acceleration time histories at each floor (channels 2-5) were used to perform the FDD output only identification. Using input case number 1 in the undamaged scenario, DC0, only four peaks appear in the singular value graph of the output PSD, as shown in Fig. 7.

In Fig. 8, the resulting mode shape vectors can be identified as, from left to right: 1) the first weak bending mode, 2) the second weak bending mode, 3) the third weak bending mode, and 4) a coupled fourth weak bending mode and first torsion mode. The first three modes coincide with the numerical model's mode shapes; however, the fourth mode differs. Unlike the numerical model's fourth mode which was a pure fourth bending mode in the weak direction, the identified mode shows a coupling with the first torsion mode. Both strong axis accelerometers (channels 6 and 7) as well as the redundant 4th story sensor in the weak direction (channel 8) confirmed that the coupled forth mode contains the first torsional component.

Once the structure is identified in the benchmark phase, damage is introduced and the model is subjected again to the input acceleration time histories. The first damage scenario is a $66 \%$ reduction of cross-sectional area of one column between the second and third floor (DC1). When identifying the structure in the DC1 state by FDD, a 

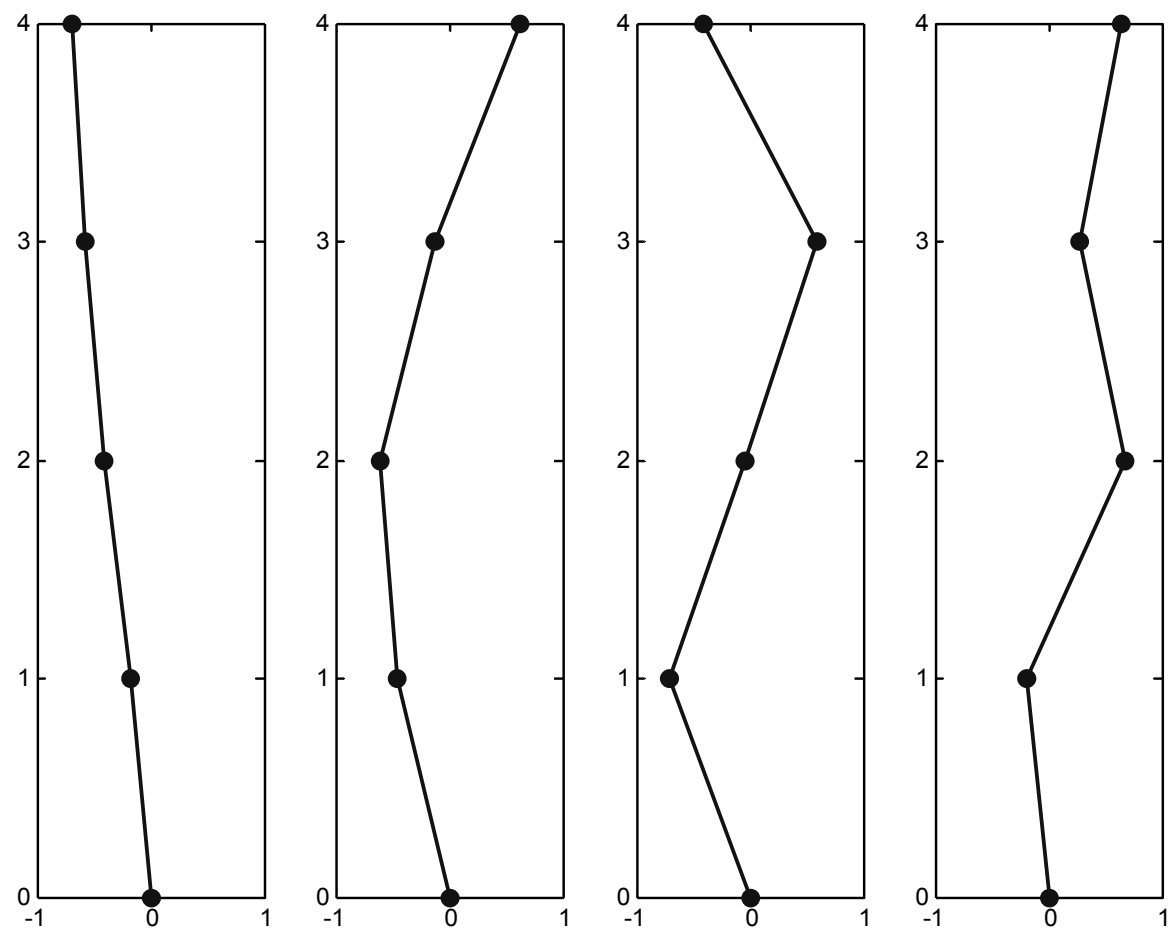

Fig. 8. Identified mode shapes, DC0 (FDD).

fifth peak clearly appears near $24 \mathrm{~Hz}$ in the dominant singular value plot, Fig. 9. The five resulting mode shapes corresponding to the five singular values are shown in Fig. 10. The first four modes are clearly the four bending modes in the weak direction, while the fifth mode appears to be a torsional mode. In the undamaged structure, the fourth mode was a coupled bending-torsional mode; the introduction of damage in the structure has decoupled this mode. Again, sensors \#6-8 verify that the fifth modal vector is indeed torsion. It should be noted that the torsional mode vector is not orthogonal to the remaining set; i.e. the fifth mode can be represented by a linear combination of the first and fourth modal vectors. Furthermore, the fourth and fifth mode vectors are geometrically similar. Therefore, it was necessary to choose a higher MAC of 0.95 to ensure that the algorithm distinguish between the two peaks' mode shape vectors.

Since the stiffness reduction of DC1 is quite significant, a further damage scenario with a $33 \%$ cross section reduction was also studied (DC2). For input case 1, the FDD algorithm yielded a dominant singular value plot that shows again five peaks, similar to DC1 (Fig. 11). Even though the stiffness reduction in the damaged column is less severe for DC2 than for DC1, the decoupling of the fourth bending mode and the first torsional mode still appears clearly on the dominant singular value plot. The five peaks correspond to five mode shapes which are essentially identical to those obtained in the previous damage scenario (Fig. 10). The strong similarity between the singular value plots of DC1 and DC2 provides evidence that the model's output spectral density function reacts to any significant damage that causes structural asymmetry by causing the torsional mode to move to the right in the frequency spectrum and gain significantly in magnitude.

For all 15 cases, the FDD algorithm identified the natural frequencies and damping ratios of the structure, as outlined in Table 5a and Fig. 12 for all three damage scenarios. The solid bars signify a confidence range of one standard deviation from the mean of the identified quantity while the error bars show the extreme value range for each quantity. As expected, the FDD algorithm provides a precise identification of the structure's natural frequencies for all ground excitation cases. The identified frequencies are stable with respect to both the magnitude and spectral content of the input excitation used to perform the identification.

Considering the results shown in Fig. 12, a clear relationship between the identified frequencies and damage becomes evident. The change in stiffness due to damage for each frequency is shown in Fig. 13. The torsional mode 


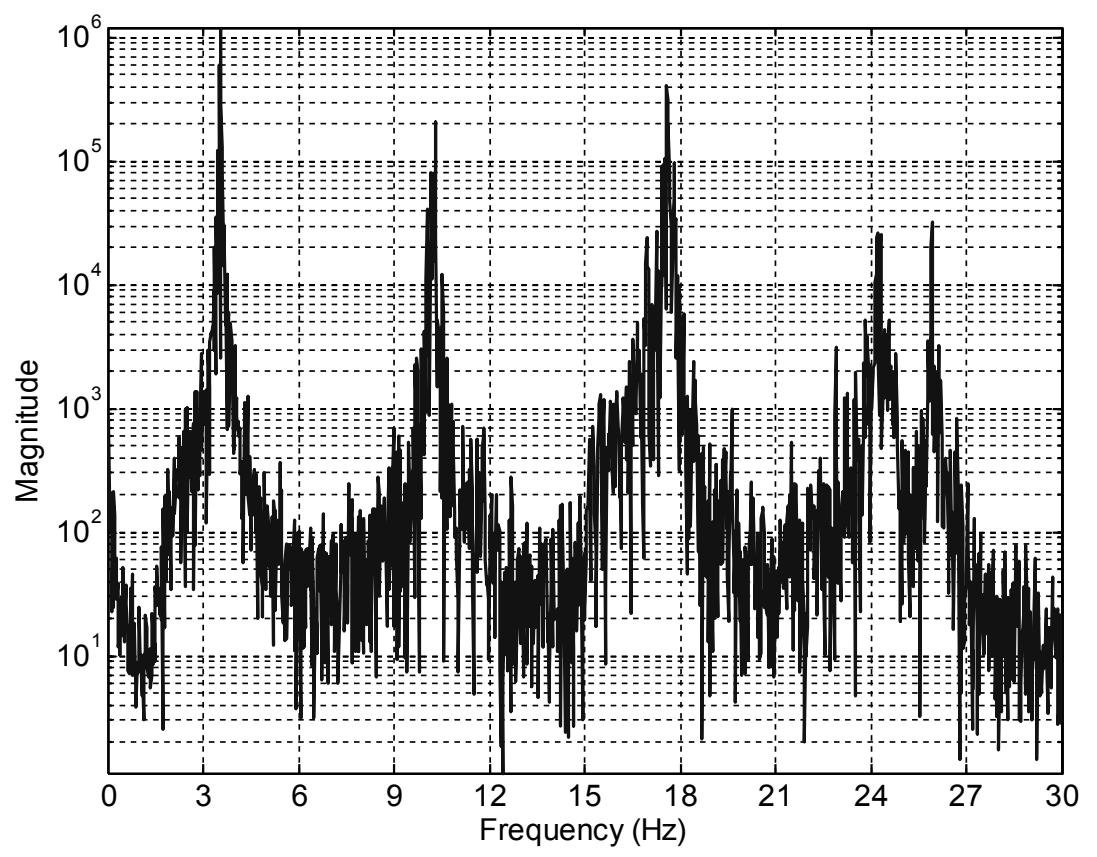

Fig. 9. Dominant singular value for output PSD, DC1 (FDD).
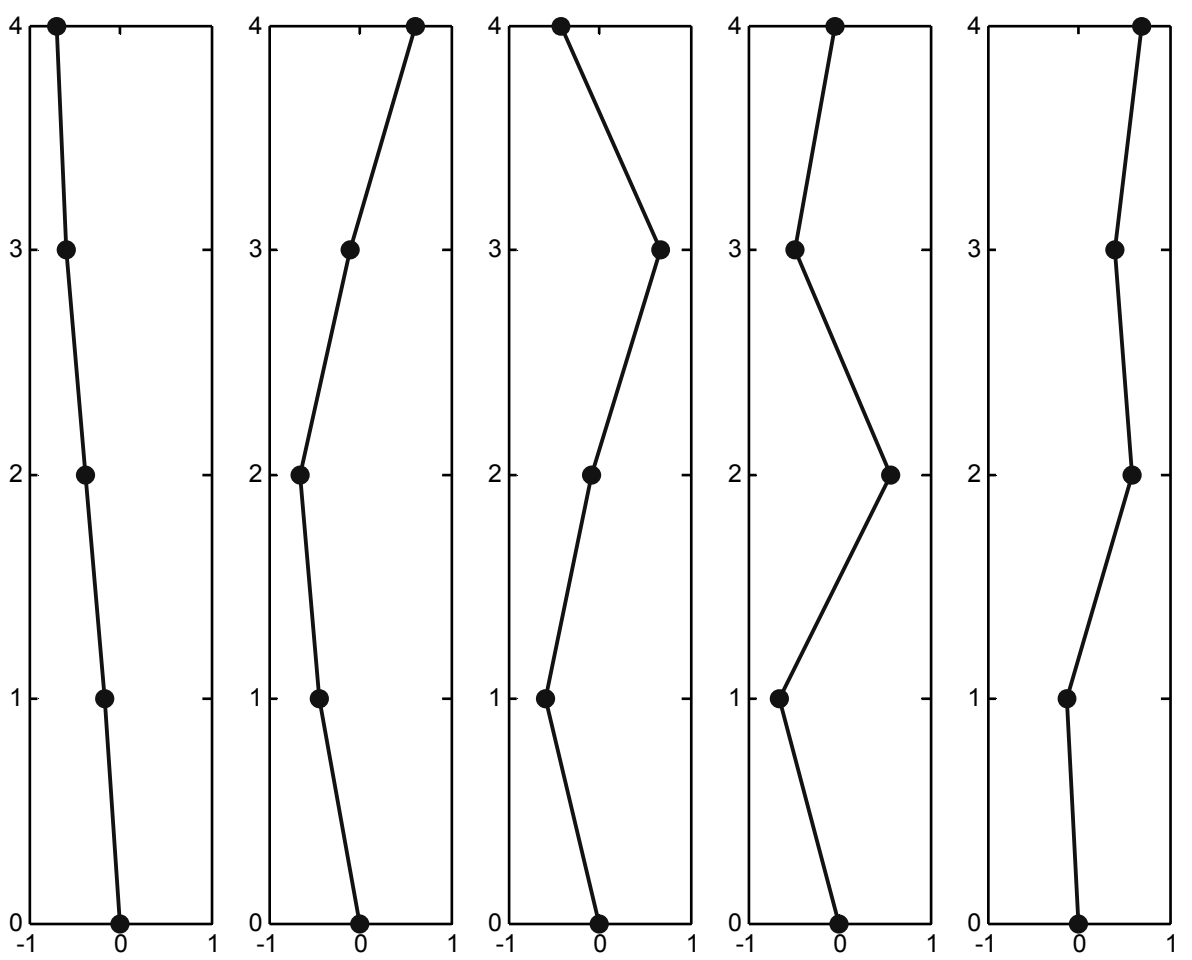

Fig. 10. Identified mode shapes, DC1 (FDD).

is not listed here since it cannot be detected independently from the fourth bending mode in the undamaged state of the model. The fourth frequency shows the strongest response to damage with a $6.07 \%$ reduction from DC0 to DC1, 
Table 5

Identified frequencies (a) and damping ratios (b) of physical model by FDD

\begin{tabular}{crrr} 
Mode & \multicolumn{3}{c}{ Identified Frequency (Hz) by FDD } \\
& DC0 & DC1 & \multicolumn{1}{c}{ DC2 } \\
1 & $3.69 \pm 0.05$ & $3.53 \pm 0.03$ & $3.68 \pm 0.04$ \\
2 & $10.62 \pm 0.10$ & $10.24 \pm 0.04$ & $10.52 \pm 0.05$ \\
3 & $18.25 \pm 0.08$ & $17.58 \pm 0.04$ & $18.08 \pm 0.08$ \\
4 & $25.71 \pm 0.20$ & $24.15 \pm 0.05$ & $24.97 \pm 0.09$ \\
5 & - & $25.90 \pm 0.13$ & $25.94 \pm 0.19$
\end{tabular}

(a)

\begin{tabular}{cccc} 
Model & \multicolumn{3}{c}{ Identified Damping (\%) by FDD } \\
& DC0 & DC1 & DC2 \\
1 & $0.99 \pm 0.11$ & $0.84 \pm 0.14$ & $0.98 \pm 0.38$ \\
2 & $0.38 \pm 0.07$ & $0.44 \pm 0.09$ & $0.37 \pm 0.12$ \\
3 & $0.30 \pm 0.04$ & $0.26 \pm 0.04$ & $0.29 \pm 0.04$ \\
4 & $0.13 \pm 0.04$ & $0.20 \pm 0.03$ & $0.17 \pm 0.03$ \\
5 & - & $0.17 \pm 0.04$ & $0.14 \pm 0.03$
\end{tabular}

(b)

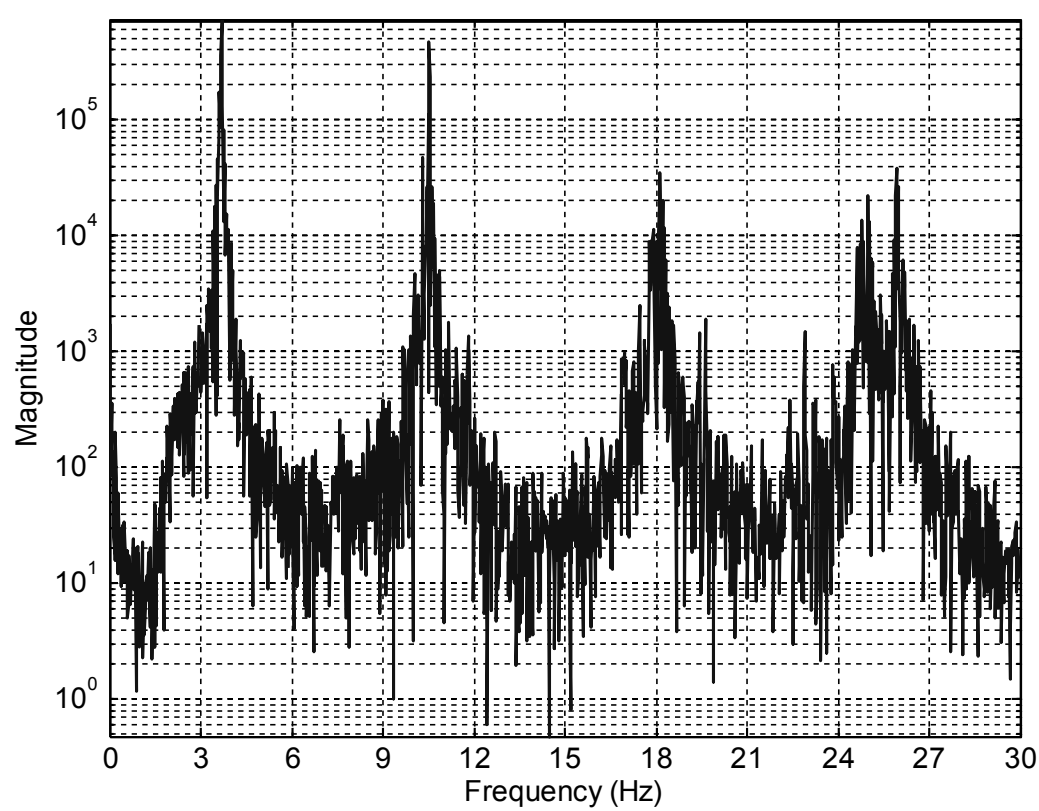

Fig. 11. Dominant singular value for output PSD, DC2 (FDD).

and $2.88 \%$ from DC0 to DC2. Less evident, but still easily recognizable is the change in the other three frequencies. However, this is expected since the interstory stiffness reductions are relatively high $-22.2 \%$ for DC1 and $13.9 \%$ for DC2 - and the frequency changes are detectable, but not of the same magnitude as the local damage. The identified frequencies can be used as a scalar indicator for the presence of damage in a structure, but it is not possible to establish either the damage location or its magnitude. All tests were conducted in the same environmental conditions, i.e. constant temperature, but in a real-life structure, temperature fluctuations and other environmental variables may easily mask a frequency change of this magnitude $[25,26]$. These findings confirm the usual understanding that in civil engineering structures - e.g. building and bridges - natural frequencies are not very sensitive to damage and should not be considered as the sole indicator for damage. Therefore, the FDD algorithm provides little potential for damage assessment, even though it is capable in identifying closely-spaced natural frequencies and their physical mode shapes. 


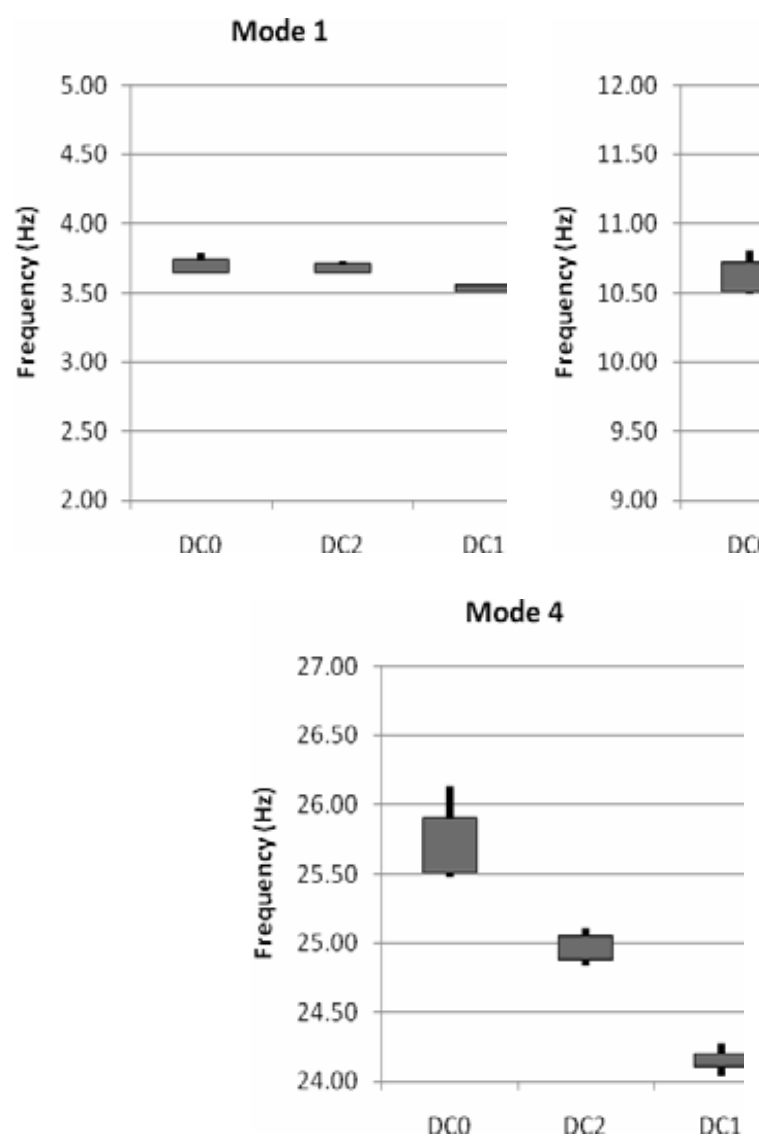

Mode 2

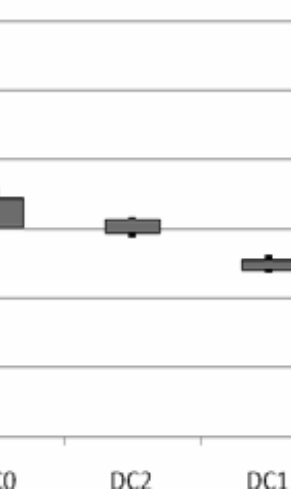

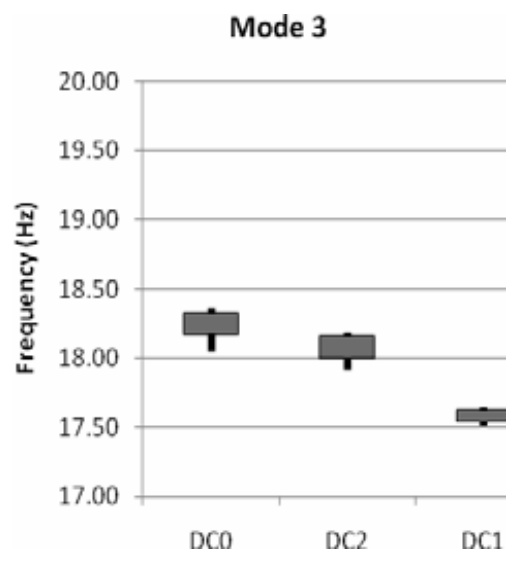

Mode 5

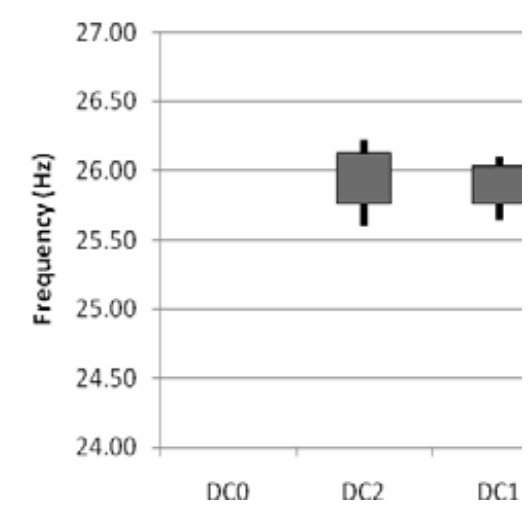

Fig. 12. Evolution of identified frequencies due to damage (FDD) ; solid bars signify $\mu \pm \sigma$ range and error bars signify minimum and maximum values.

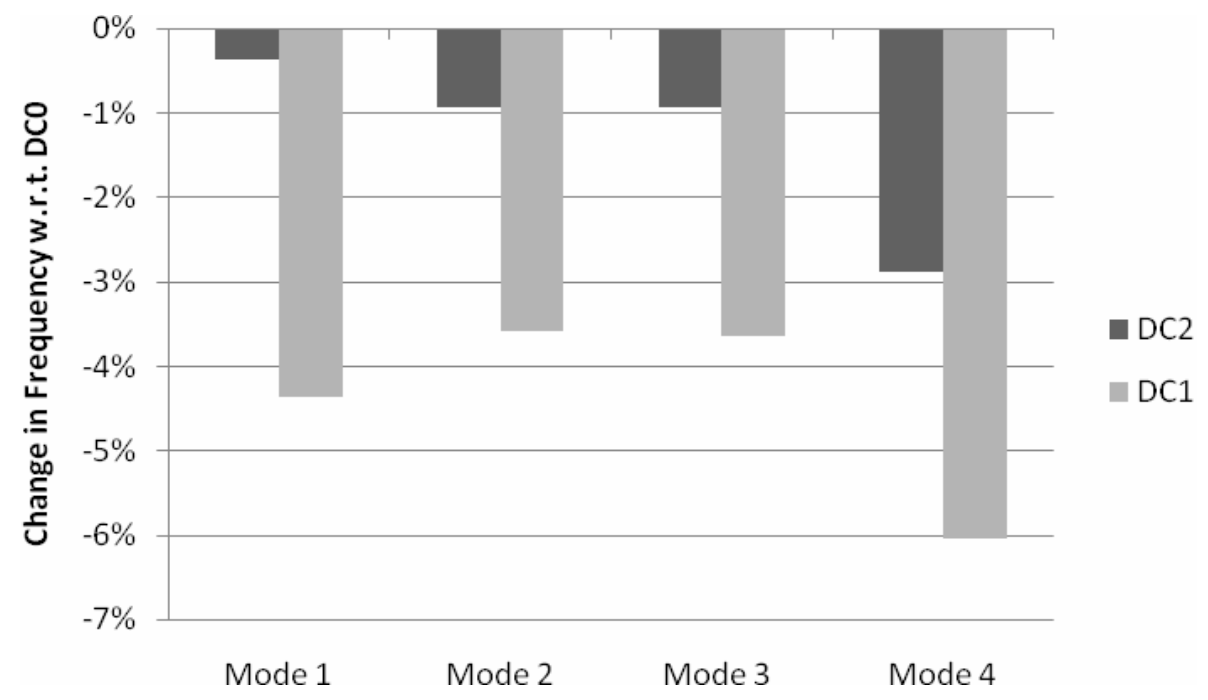

Fig. 13. Change in identified modal frequencies due to damage (FDD). 

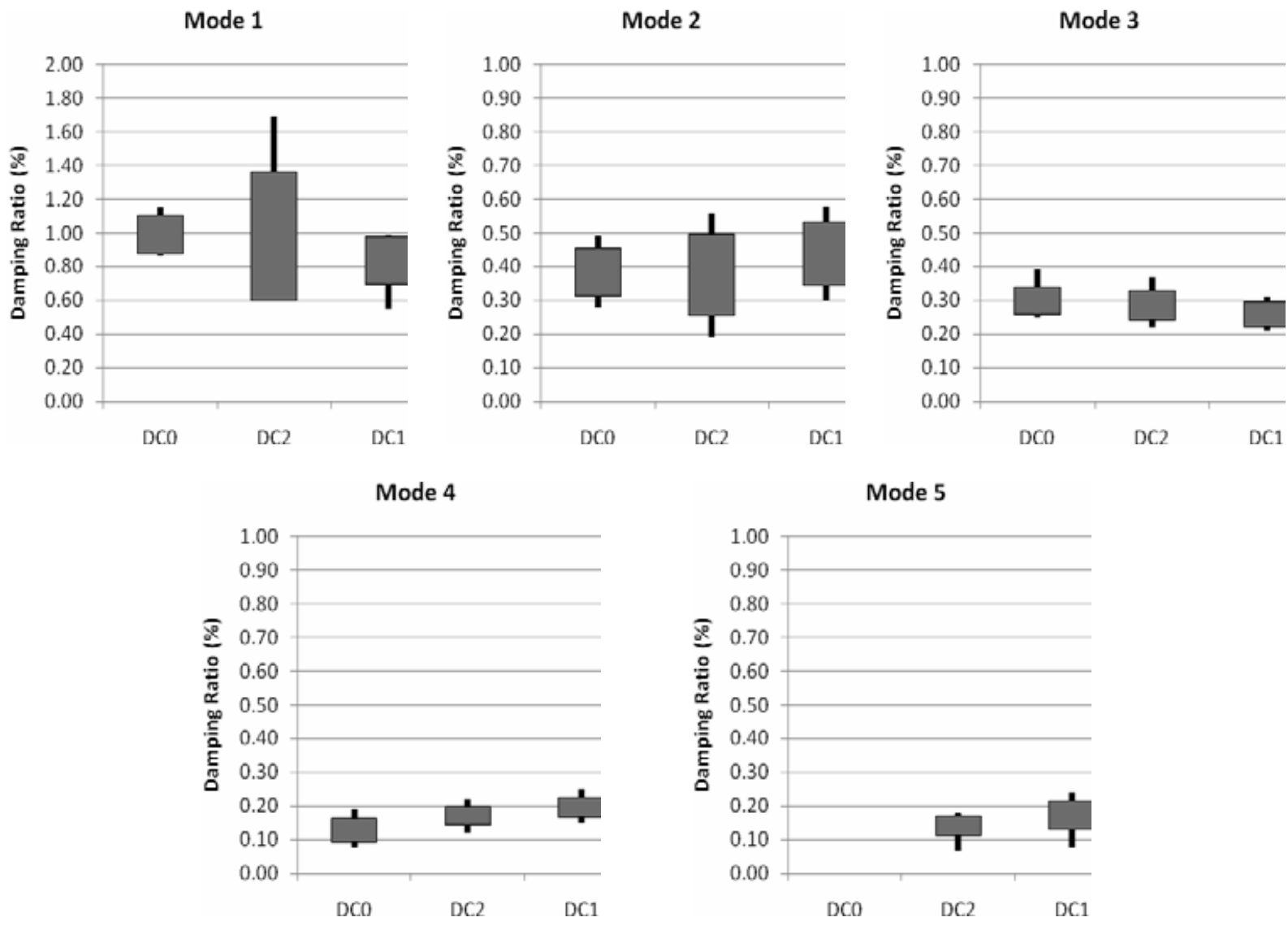

Fig. 14. Evolution of identified damping ratios due to damage (FDD) ; solid bars signify $\mu \pm \sigma$ range and error bars signify minimum and maximum values.

Unlike the identified frequencies, the identified modal damping ratios show a strong dependence on the type of input time history used to excite the structure, as outlined in Table $5 \mathrm{~b}$ and Fig. 14. In all damage scenarios, the identified damping ratio performed with the band-limited white noise (run cases \#13-15) deviates below the mean by one standard deviation, while the identification by the Northridge and Kobe earthquakes deviates above the mean by approximately one standard deviation. By inspection (Fig. 3) the band-limited white noise time history contains, by design, significantly more power above $10 \mathrm{~Hz}$ than the earthquake time histories. Also, while the El Centro and Hachinohe earthquakes contain the majority of their spectral power in the range of $0-10 \mathrm{~Hz}$, the majority of the spectral power of the Northridge and Kobe earthquakes is limited to the range of $0-5 \mathrm{~Hz}$. There exists an inverse relationship between the upper bound of the frequency content and the value of the identified damping. An identification using excitation with significant power in low frequency causes the damping estimation to be high while a broad-banded excitation with spectral power in higher frequencies causes the damping to be underestimated.

The average modal damping ratios for the three damage scenarios seem to follow no clear trend (Fig. 15). The identified damping ratio of the first and third modes decreases once damage is induced while the damping ratio for the second and fourth modes increase. Furthermore, the variation in damping ratios between input cases is too large for them to serve as a reliable damage indicator, even if a global trend were apparent. A simple spectral analysis of the structure's response, undamaged vs. damaged, has confirmed that the higher modes increase in magnitude when damage occurs. As a matter of fact, the first torsional mode's energy is intertwined with the fourth bending mode in the weak direction; hence, the increase in the magnitude of the PSD associated with the torsional mode due to asymmetry in the damaged structure also spurs an increased activation of the fourth weak bending mode. Physically, a decrease in the first and an increase in the fourth mode's damping factor may be explained by this increased activation of the higher modes once damage is introduced. 
Table 6

Identified frequencies (a) and damping ratios (b) of physical model by OKID

\begin{tabular}{crrr} 
Mode & \multicolumn{3}{c}{ Identified Frequency $(\mathrm{Hz})$ by OKID } \\
& DC0 & DC1 & DC2 \\
1 & $3.68 \pm 0.03$ & $3.54 \pm 0.01$ & $3.66 \pm 0.02$ \\
2 & $10.60 \pm 0.09$ & $10.20 \pm 0.02$ & $10.50 \pm 0.04$ \\
3 & $18.21 \pm 0.07$ & $17.55 \pm 0.02$ & $18.03 \pm 0.05$ \\
4 & $25.81 \pm 0.12$ & $24.14 \pm 0.03$ & $24.91 \pm 0.07$ \\
5 & - & $25.89 \pm 0.07$ & $25.95 \pm 0.08$
\end{tabular}

(a)

\begin{tabular}{cccc} 
Mode & \multicolumn{3}{c}{ Identified Damping (\%) by OKID } \\
& DC0 & DC1 & DC2 \\
1 & $0.95 \pm 0.19$ & $0.90 \pm 0.08$ & $0.94 \pm 0.14$ \\
2 & $0.51 \pm 0.11$ & $0.43 \pm 0.06$ & $0.52 \pm 0.11$ \\
3 & $0.47 \pm 0.10$ & $0.34 \pm 0.05$ & $0.48 \pm 0.09$ \\
4 & $0.61 \pm 0.17$ & $0.37 \pm 0.05$ & $0.59 \pm 0.16$ \\
5 & - & $0.47 \pm 0.07$ & $0.49 \pm 0.17$
\end{tabular}

(b)

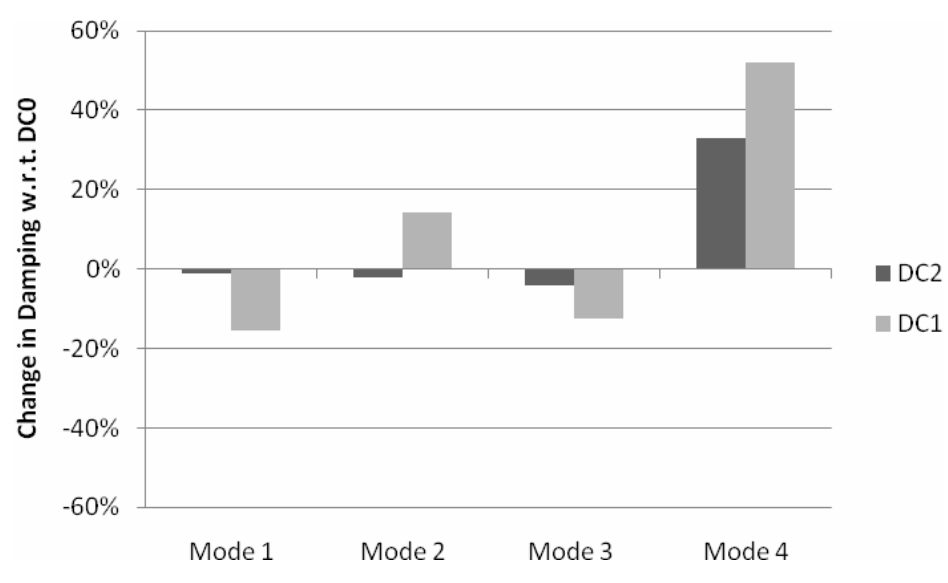

Fig. 15. Change in identified modal damping ratios due to damage (FDD).

Many procedures have been proposed for damage identification using information about mode shapes and their derivatives. It has been demonstrated that the comparison of mode shapes in different structural configurations does not reach the desired goal. Better results can be reached by using, for example, mode shape curvatures. However, a significantly larger number of sensors would be required in order to obtain numerically stable mode shape curvatures for the different scenarios [27].

Since the FDD algorithm does not provide orthogonal complex eigenvectors, a physical parameter identification for mass, damping, and stiffness matrices cannot be performed.

\subsection{ERA/OKID identification}

The input and response acceleration time histories obtained from the shake table tests were also used to identify the structure with the ERA/OKID algorithm. The ERA/OKID algorithm requires the user to choose the number of significant Hankel singular values to be used in the pseudoinverse of said matrix. For the undamaged scenario, DC0, four singular value pairs dominate the spectrum. Consequently, the order of the minimal realization is fixed at the first four singular value complex conjugate pairs.

The natural frequencies identified by the algorithm are shown in Table 6a and Fig. 16. Examining the trends of the average values of natural frequencies for each damage case, some conclusions about global trends due to damage 

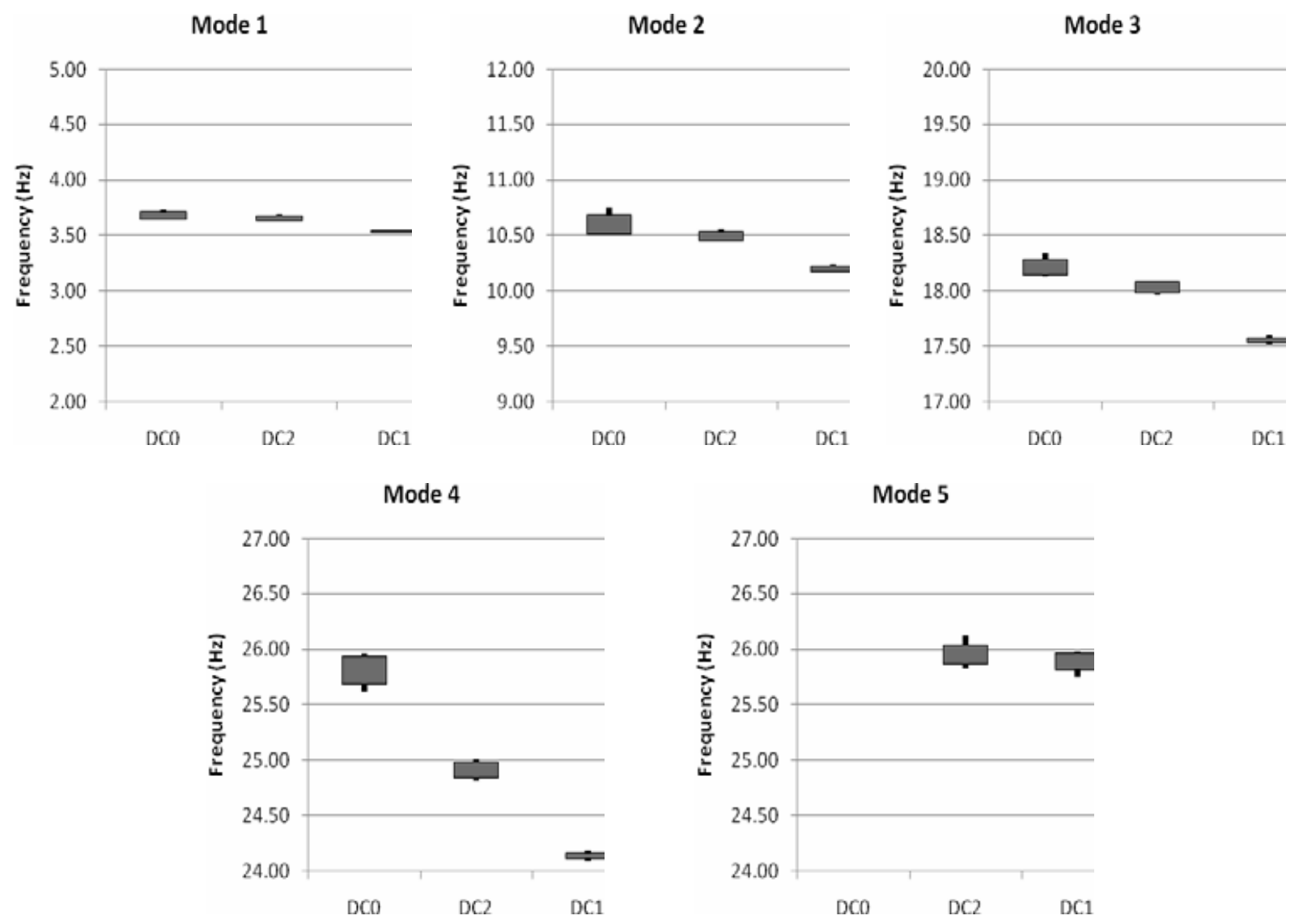

Fig. 16. Evolution of identified frequencies due to damage (OKID); solid bars signify $\mu \pm \sigma$ range and error bars signify minimum and maximum values.

can be made. The results for all three damage scenarios are highly stable, with the notable exception of the fifth natural frequency. The OKID (Fig. 17) and FDD (Fig. 13) algorithms provide practically identical results for the identified frequencies. Again, the most evident reduction in frequency occurs in the highest mode, showing a $6.47 \%$ and $3.49 \%$ reduction in frequency for DC1 and DC2, respectively. This finding supports previous studies, which have shown that higher order modes exhibit significantly higher mobility with respect to changes in the structure's stiffness [28]. In civil engineering structures, predominant modes are usually in the low-frequency range (below $1 \mathrm{~Hz}$ ), and this makes frequencies not particularly sensitive to structural damage. However, this observation should not be generalized since different damage scenarios might affect differently the various mode shapes. As an example, the first bending mode has most of its stress in the cantilever root, so damage at the base of a beam-like structure will induce a stronger change in the fundamental mode than in higher modes. Hence, it is advisable not to rely only on information from natural frequencies as indicators of structural damage.

Similar to the damping ratios identified by the FDD algorithm, the variation of the ratios with respect to the type of input is very strong, as shown in Table $6 \mathrm{~b}$ and Fig. 18. Again, the identified modal damping factors from the band-limited white noise (trials 13 to 15 ) are significantly lower than the damping identified through earthquake time history inputs. The identified frequencies and damping coefficients are close to the results obtained through FDD. As previously discussed, the broader spectral content of the band-limited white noise time history, especially above $10 \mathrm{~Hz}$, appears to cause the structure to exhibit lower modal damping. As with the damping identified with FDD, no clear trend is apparent between modal damping and induced damage for the data set generated by OKID, Fig. 19.

A numerical sensitivity analysis was performed with a 3D finite element model of the structure. The model was populated with the exact dimensions of the test structure and all three damage case geometries - DC0, DC1, and DC2 - underwent an eigenvalue analysis. The results compare very well to the experimental data, Table 7 . The 


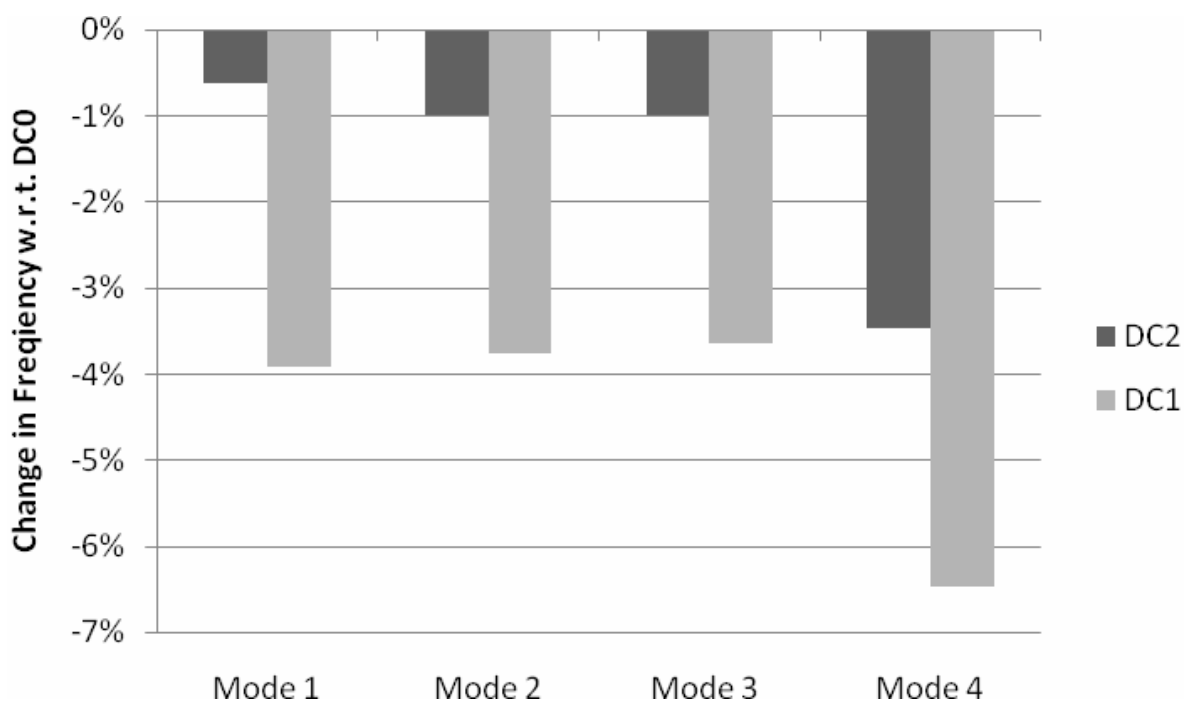

Fig. 17. Change in identified modal frequencies due to damage (OKID).

Mode 1

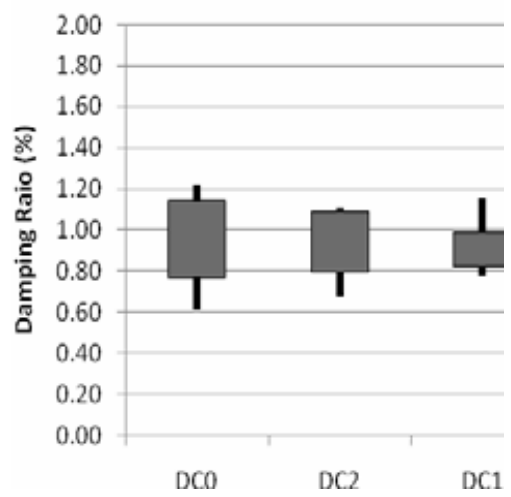

Mode 2

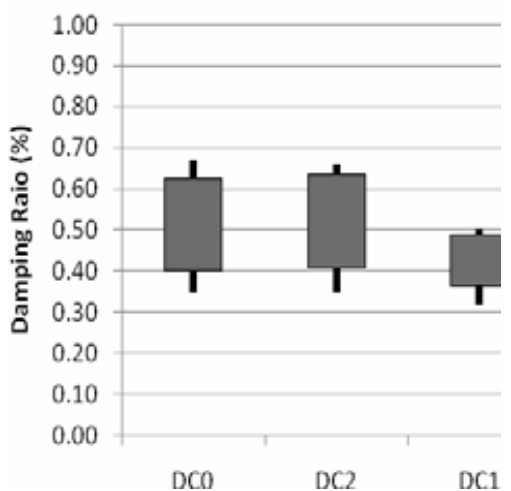

Mode 3

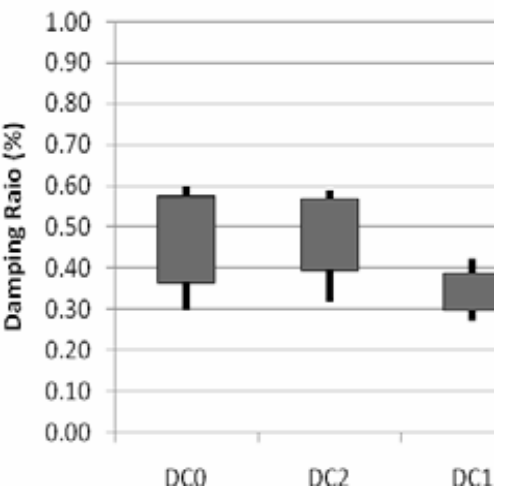

Mode 4
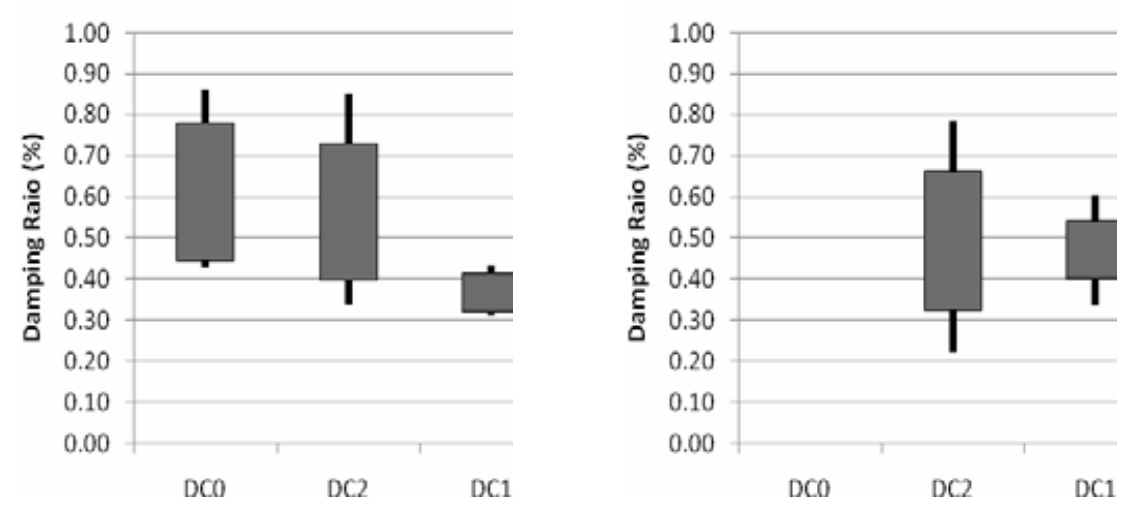

Fig. 18. Evolution of identified damping factors due to damage (OKID) ; solid bars signify $\mu \pm \sigma$ range and error bars signify minimum and maximum values. 
Table 7

Natural frequency changes of FEM due

to simulated damage

$\begin{array}{ccc}\text { Mode } & \mathrm{DC} 1 \Delta \mathrm{F}(\%) & \mathrm{DC} 2 \Delta \mathrm{F}(\%) \\ 1 & -0.90 & -0.49 \\ 2 & -2.29 & -1.29 \\ 3 & -2.88 & -1.64 \\ 4 & -6.40 & -3.72\end{array}$

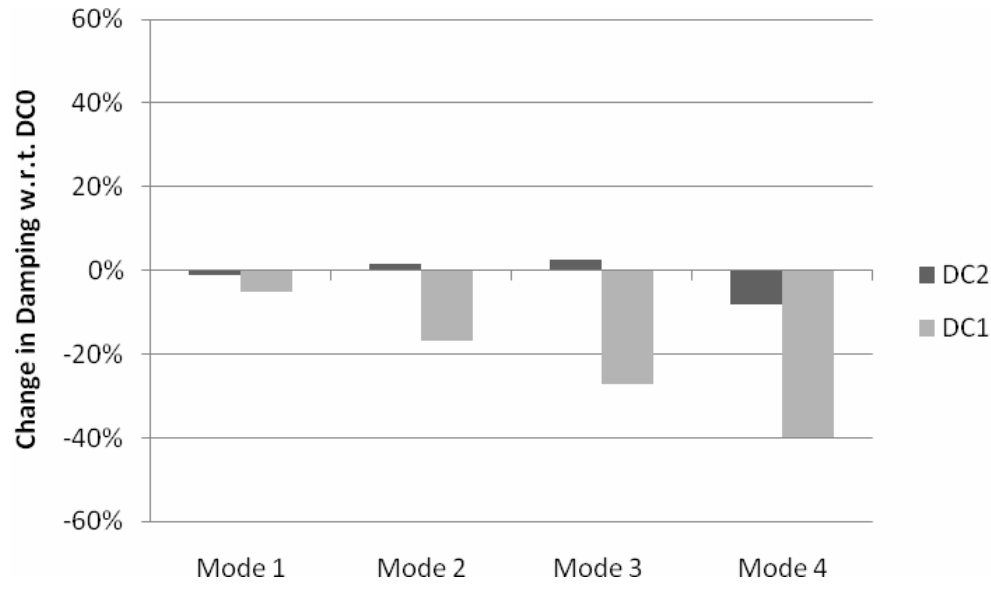

Fig. 19. Change in identified modal damping ratios due to damage (OKID).

changes in frequency of the fourth mode coincide almost exactly with the values identified by both FDD and OKID. Overall, these numerical results support the trend that was observed in the experimental data, i.e. a relatively strong response of the higher modes to the induced damage when compared to the lower ones.

Interestingly, the OKID (Fig. 19) and FDD (Fig. 15) algorithms produce different results with respect to identified modal damping ratios. While the results from FDD show an increase in damping of the higher order modes and a decrease of the lower order modes when damage is introduced, the OKID results show a general decrease in damping ratios for all modes. However, this trend is very weak, especially in the lower order modes.

\subsection{MLK identification}

In the case of the ERA/OKID algorithm, the identified complex eigenvectors can be used to obtain the physical parameters of the system, most importantly the system's stiffness matrix. The evolution of the stiffness matrix provides a very intuitive representation of the structure's overall condition. A benchmark structure can be defined in this framework, using the structure's identified stiffness matrix as an indicator of possible structural damage. Since the experimental structure in question is fully instrumented (i.e. at least one sensor per degree of freedom in the direction of excitation), the identified stiffness matrix has physical significance, representing the values of the classical interstory stiffness coefficients of a shear building, $k_{i}$.

In order to provide a more stable identification of the stiffness matrix, the masses of all degrees of freedom were assumed to be known and defined at $37 \mathrm{~kg}$. Using the known diagonal mass matrix and the complex eigenvectors from the OKID identification, the MLK algorithm identified the stiffness matrix, $K_{D C \#}$, for the undamaged case:

$$
K_{D C 0}=\left[\begin{array}{cccc}
5.94 & -3.43 & 0.83 & -0.14 \\
-3.43 & 5.02 & -2.75 & 0.40 \\
0.83 & -2.75 & 4.21 & -2.03 \\
-0.14 & 0.40 & -2.03 & 1.67
\end{array}\right](\mathrm{N} / \mathrm{m})
$$

Even though the matrix is not purely tridiagonal, as would be expected for the stiffness matrix of a shear type structure, the three-diagonal terms still dominate the matrix. Looking at the various identifications for the different 
input-output sets, it is possible to analyze the variability of the identified stiffness through the coefficients of variation, $c_{V}^{D C \#}$, for the elements of the stiffness matrix:

$$
c_{V}^{D C 0}=\left[\begin{array}{cccc}
0.76 & 2.74 & 2.75 & 16.17 \\
2.74 & 2.46 & 2.29 & 6.73 \\
2.75 & 2.29 & 1.35 & 1.33 \\
16.17 & 6.73 & 1.33 & 1.64
\end{array}\right](\%)
$$

The stiffness matrix, when identified with known nodal masses, proves to be quite robust, varying only as much as $2.7 \%$ in the tridiagonal region of the matrix. The values located far off the diagonal tend to exhibit higher variation; however, these terms are usually of smaller magnitude and represent pure numerical error from the identification routine.

For damage cases DC1 and DC2, the MLK algorithm provides the following stiffness matrices:

$$
\begin{aligned}
K_{D C 1} & =\left[\begin{array}{cccc}
5.75 & -3.05 & 0.61 & -0.21 \\
-3.05 & 4.18 & -2.18 & 0.38 \\
0.61 & -2.18 & 3.60 & -1.83 \\
-0.21 & 0.38 & -1.83 & 1.56
\end{array}\right](N / m) \\
K_{D C 2} & =\left[\begin{array}{cccc}
5.99 & -3.28 & 0.81 & -0.25 \\
-3.28 & 4.63 & -2.55 & 0.44 \\
0.81 & -2.55 & 4.00 & -1.97 \\
-0.25 & 0.44 & -1.97 & 1.65
\end{array}\right](N / m)
\end{aligned}
$$

When considering the variation of the stiffness coefficients with respect to the type of input excitation, it becomes apparent that the identification of the stiffness matrix is also robust with respect to excitation type in the damaged state of the structure:

$$
\begin{aligned}
c_{V}^{D C 1} & =\left[\begin{array}{cccc}
1.06 & 1.77 & 7.44 & 12.08 \\
1.77 & 1.31 & 4.66 & 5.87 \\
7.44 & 4.66 & 3.07 & 2.10 \\
12.08 & 5.87 & 2.10 & 1.67
\end{array}\right](\%) \\
c_{V}^{D C 2} & =\left[\begin{array}{cccc}
0.25 & 0.88 & 4.84 & 16.13 \\
0.88 & 0.97 & 1.24 & 7.16 \\
4.84 & 1.24 & 0.80 & 0.79 \\
16.13 & 7.16 & 0.79 & 0.63
\end{array}\right](\%)
\end{aligned}
$$

Since both the benchmark stiffness matrix as well as the stiffness matrices for the two damage cases have been accurately computed, it is possible to calculate the changes in stiffness, $\Delta \mathrm{K}$, for each damage scenario:

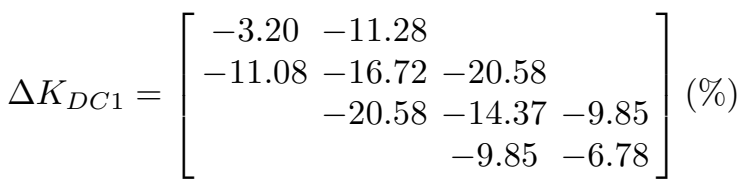

$$
\begin{aligned}
& \Delta K_{D C 2}=\left[\begin{array}{crrr}
0.84 & -4.37 & & \\
-4.37 & -7.70 & -7.45 & \\
& -7.45 & -4.95 & -2.80 \\
& & -2.80 & -1.14
\end{array}\right](\%)
\end{aligned}
$$

In both damage cases, the stiffness matrix shows significant local deviations from the benchmark structure. Not only can the amount of damage be detected quite easily, but also its location; in fact, looking at the position of greatest stiffness change allows us to pinpoint where structural damage has occurred and quantify its magnitude. The identified stiffness matrices show a clear concentration of stiffness reduction in the coefficient $k_{3}$, the interstory 
stiffness between the second and third floors of the structure. The identified stiffness reductions of $20.58 \%$ and $7.45 \%$ for DC1 and DC2, respectively, correlate quite well to the theoretical stiffness reductions of $22.2 \%$ and $13.9 \%$. The discrepancies can be explained by the fact that, in calculating the theoretical interstory stiffness, the floor plates were assumed to be completely rigid and the diagonal bracing was assumed to provide no bending stiffness. The reduction of theoretical interstory stiffness may have been overestimated since the out of plane stiffness of the bracing members was ignored in the theoretical stiffness calculation.

In order to define a minimum threshold of damage detection, the coefficient of variation with respect to input type of each stiffness coefficient, $k_{i}$, being monitored for damage must be considered. In order for damage to be detected by the algorithm, the magnitude of the stiffness change, $\Delta k_{i}$, must be greater than the numerical noise of the identification routine. For the undamaged case, the greatest variation of any stiffness coefficient is $1.79 \%$ while DC1 has a maximum variation of $4.66 \%$ and DC2 $1.24 \%$. Hence, in this case, even the smaller stiffness reduction of $13.9 \%$ can easily be identified by algorithm since the variation of the governing local stiffness coefficient, $k_{3}$, lies well above the maximum coefficient of variation with respect to change in input excitation.

\section{Conclusions}

In this work three identification algorithms (the Frequency Domain Decomposition algorithm, the Observer Kalman filter Identification algorithm, and the MLK algorithm) have been tested in applications of structural health monitoring of frame structures subjected to earthquake excitations. Both FDD and OKID provide a reliable identification of the structural frequencies. However, it was shown that, although both algorithms identified the natural frequencies of the structure with negligible error, this variable is not sufficiently responsive to minor damage and can therefore not be used as a practical indicator for structural damage. In this specific case, where damage was induced between the second and third floor column of a four-story building, the lower order modal frequencies did not respond as strongly as the higher ones to the induced damage. An FEM sensitivity analysis of the natural frequencies with respect to damage (e.g. DC0 vs. DC1, DC2) underlines these observations. This conclusion about the sensitivity of the natural frequencies to the presence of damage should not be generalized since different damage scenarios might affect differently the various mode shapes.

Furthermore, both algorithms failed to identify the structure's damping to a sufficient degree of accuracy, with OKID providing a somewhat more precise identification. In both cases, the identified damping coefficients were closely coupled to the spectral content of the input excitation, negating any meaningful conclusions about the structure from the evolution of damping alone.

However, the OKID algorithm provides the complex mode shapes of the structure which, if scaled correctly, can be used to provide a physical identification of the structure through the MLK algorithm. The stiffness matrix, especially when linked to a known or an accurately estimated mass matrix, provides a very stable identification result that is robust against variation in both the magnitude and spectral content of the excitation and can be used to identify and quantify structural damage. Simulated reductions in interstory stiffness as low as $13.9 \%$ could be easily identified by the algorithm. In addition to the accurate prediction of the amount of damage, this approach allows the engineer to locate the damaged area within the structure by looking at where the changes in the stiffness matrix have occurred. However, it is important to note that this work was performed in a laboratory environment under constant climate conditions, so no climate-specific factors skewed the results of this identification.

With respect to automation, OKID/MLK lends itself quite well to integration into a live monitoring system. The required user input, namely the assumed order of the system, the Hankel singular value cutoff, and the estimated mass of the system can be defined in the algorithm once the benchmark identification has been performed. However, it would be dangerous to allow the system to automatically identify these modes without periodic validation of the identification routine by experienced personnel. As this study has shown, damage that causes geometric asymmetry in a structure may cause a torsional mode to significantly gain in dominance, possibly replacing another mode in the truncated Hankel singular value matrix. Therefore, it is very important that the user be conscious of any evolution of the modal characteristics of the system over time, i.e. the physical mode shape of each identified dominant mode in the health monitoring scheme. In this model, a torsional mode induced by damage not only migrated to a higher frequency, but also surpassed the fourth bending mode in magnitude. It is in the detection of such global changes in 
the modal spectrum that the FDD algorithm may serve as a support tool, providing the physical mode shapes to the user for inspection. Therefore, an automated OKID routine with periodic validation of the modal landscape by use of FDD can provide a powerful structural health monitoring system.

\section{References}

[1] D.J. Ewins, Modal Testing: Theory and Practice, 1984.

[2] J.E. Mottershead and M.I. Friswell, Model Updating in Structural Dynamics: A Survey, J Sound Vib 2 (1993), $347-375$.

[3] A. Berman, Mass Matrix Correction Using an Incomplete Set of Measured Modes, AIAA J 10 (1979), 1147-1148.

[4] M. Baruch, Modal Data are Insufficient for Identification of Both Mass and Stiffness Matrices, AIAA J 11 (1997), $1797-1798$.

[5] J.L. Beck and L.S. Katafygiotis, Updating Models and Their Uncertainties. I: Bayesian Statistical Framework, J Eng Mech 4 (1998), 455-461.

[6] M.S. Agbabian, S.F. Masri, R.K. Miller and T.K. Caughey, System Identification Approach to Detection of Structural Changes, J Eng Mech 2 (1991), 370-390.

[7] A. Smyth, S.F. Masri, T.K. Caughey and N.F. Hunter, Surveillance of Intricate Mechanical Systems on the Basis of Vibration Signature Analysis, ASME J Appl Mech 3 (2000), 540-551.

[8] S.R. Ibrahim and E.C. Mikulcik, A Method for the Direct Identification of Vibration Parameters From the Free Response, Shock Vib Bull 4 (1997), 183-198.

[9] S.R. Ibrahim, Random Decrement Technique for Modal Identification of Structures, J Spacecr Rockets 11 (1977), 696-700.

[10] H. Vold, J. Kundrat, G.T. Rocklin and R. Russel, A Multiple-Input Modal Estimation Algorithm for Mini Computers, SAE Trans 1 (1982), $815-821$.

[11] J.N. Juang and R.S. Pappa, An Eigensystem Realization Algorithm for Modal Parameter Identification and Model Reduction, J Guid Control Dyn 5 (1985), 620-627.

[12] J.N. Juang, J.E. Cooper and J.R. Wright, An Eigensystem Realization Algorithm Using Data Correlations (ERA/DC) for Modal Parameter Identification, Cont Theor Adv Technol 1 (1988), 5-14.

[13] J.N. Juang, M. Phan, L.G. Horta and R.W. Longman, Identification of Observer/Kalman Filter Markov Parameters: Theory and Experiments, J Guid Control Dyn 2 (1993), 320-329.

[14] H. Luş, R. Betti and R.W . Longman, Identification of Linear Structural Systems Using Earthquake-Induced Vibration Data, Earthquake Eng Struct Dyn 28 (1999), 1449-1467.

[15] H. Luş, R. Betti and R.W. Longman, Obtaining Refined First-Order Predictive Models of Linear Structural Systems, Earthquake Eng Struct Dyn 31 (2002), 1413-1440.

[16] A. Sestieri and S.R. Ibrahim, Analysis of Errors and Approximations in the Use of Modal Coordinates, J Sound Vib 2 (1994), $145-157$.

[17] D.-H. Tseng, R.W. Longman and J.N. Juang, Identification of the Structure of the Damping Matrix in Second Order Mechanical Systems, Adv Astronaut Sci 87 (1994), 166-190.

[18] M. Imregun and D.J. Ewins, Realization of Complex Modeshapes, Proceedings of the 11th International Modal Analysis Conference, Society for Experimental Mechanics, 1993, 1303-1309.

[19] S.R. Ibrahim, Computation of Normal Modes From Identified Complex Modes, AIAA J 3 (1983), $446-451$.

[20] K.F. Alvin, Second-Order Structural Identification Via State Based System Realizations, Ph.D. Dissertation, University of Colorado, 1993.

[21] M. De Angelis, H. Luş, R. Betti and R.W. Longman, Extracting Physical Parameters of Mechanical Models From Identified State Space Representations, J App Mech 69 (2002), 617-625.

[22] R. Brincker, L. Zhang and P. Andersen, Modal identification of output-only systems using frequency domain decomposition, Smart Mater Struct 10 (2001), 441-445.

[23] R. Brincker, C.E. Ventura and P. Andersen, Damping Estimation by Frequency Domain Decomposition, IMAC-XIX: A Conference on Structural Dynamics 1 (2001), 698-703.

[24] T. Ting, T.L.C. Chen and W. Twomey, Correlating mode shapes based on the modal assurance criterion, Finite Elements in Analysis and Design 14 (1993), 353-360.

[25] A. Asghari, E. Johnson and R. Nigbor, Environmental Effects on Identified Parameters of the NEES SFSI Test Structure, Proceedings of the 4th World Conference on Structural Control and Monitoring (2006).

[26] N. Londoño, D. Lau, Uncertainties in Structural Health Monitoring, Proceedings of the 4th World Conference on Structural Control and Monitoring (2006).

[27] D. Zonta, D. Bernal, O. Bursi, M. Pozzi and O. Tigli, Localization of Damage in a Set of Concrete Beams: Comparison of Two Techniques, Proceedings of the 4th World Conference on Structural Control and Monitoring (2006).

[28] J. Caicedo, A. Dutta and B. Zarate, System Identification and Model Updating of the Bill Emerson Memorial Bridge, Proceedings of the 4th World Conference on Structural Control and Monitoring, (2006). 

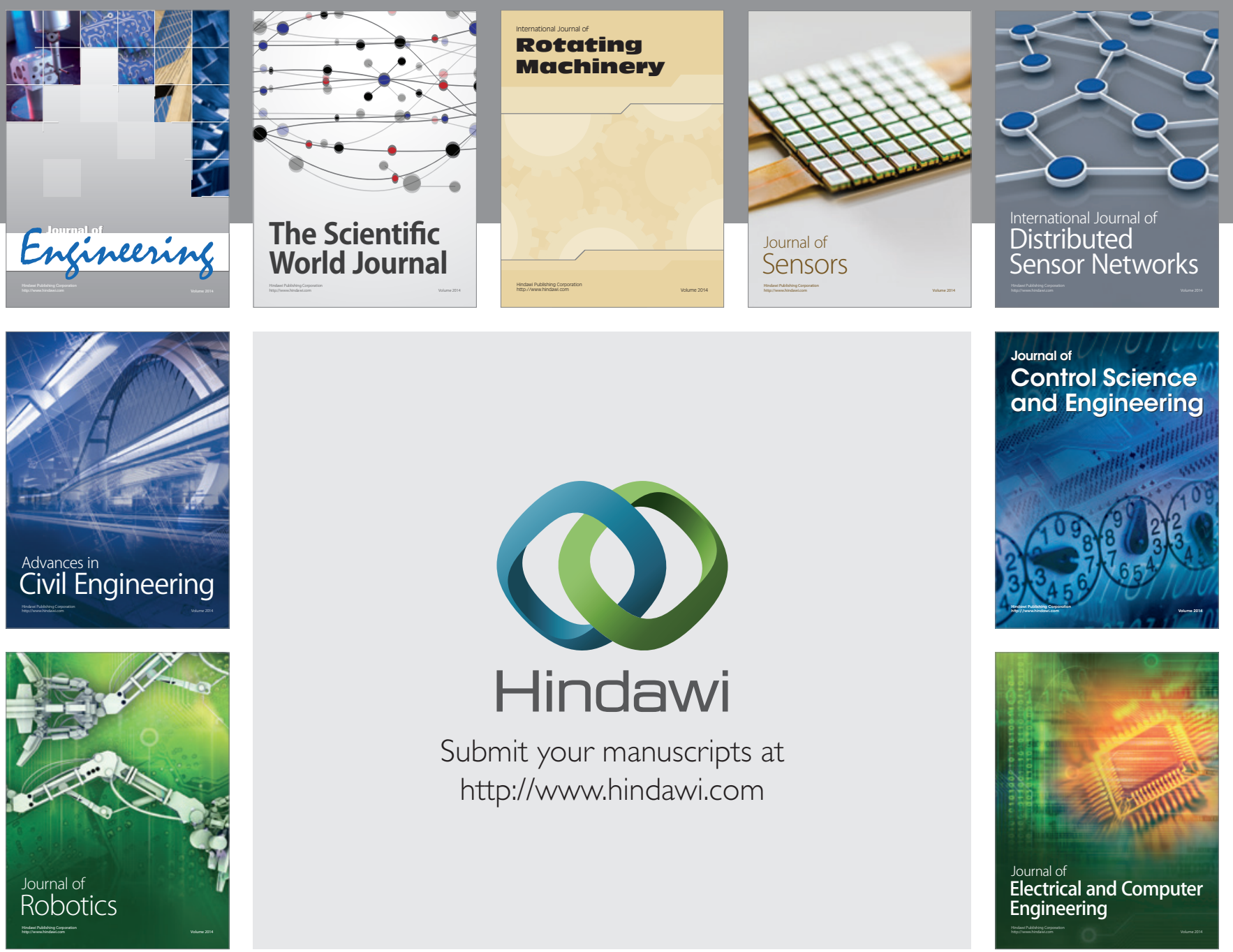

Submit your manuscripts at

http://www.hindawi.com
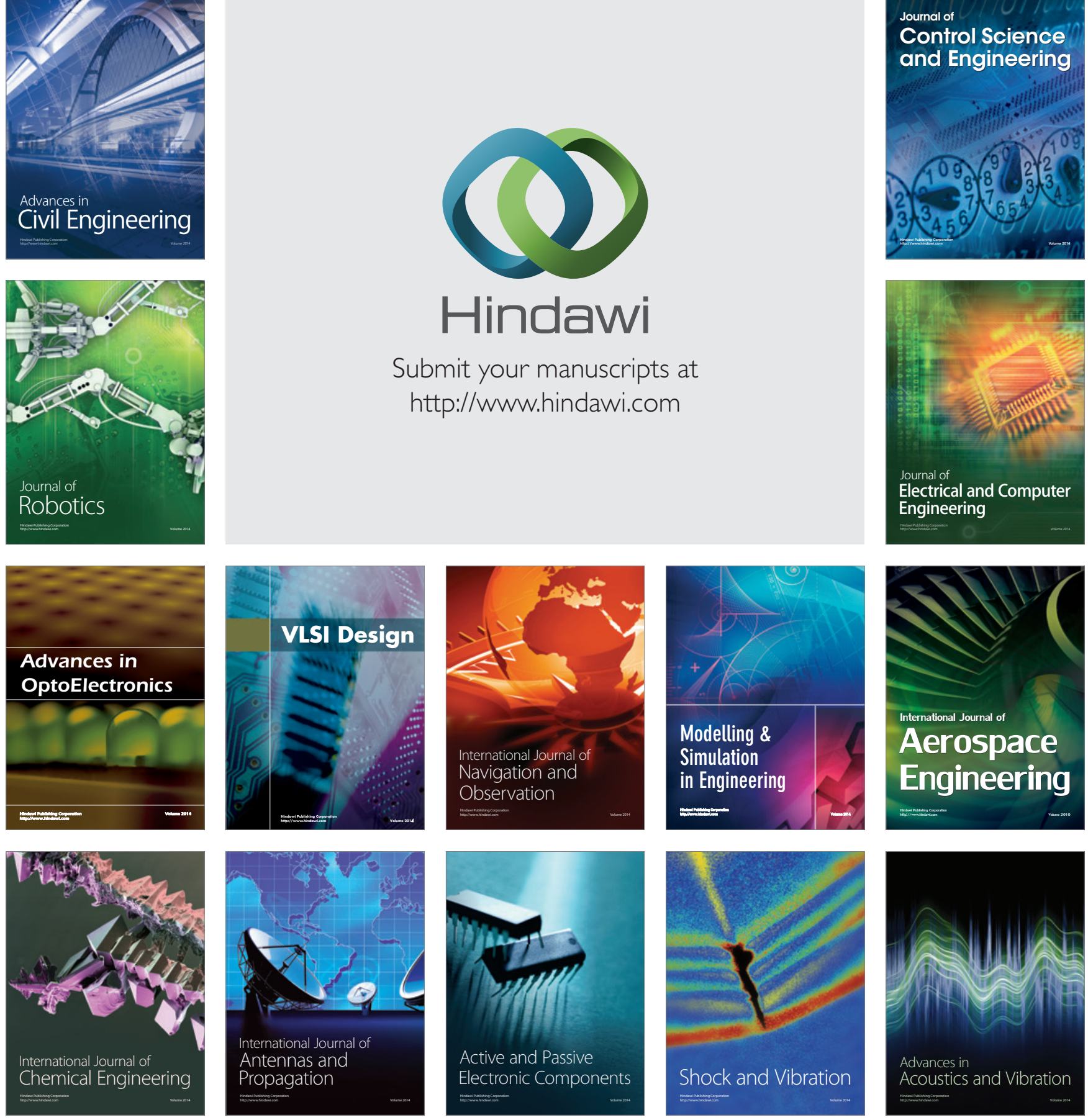